\title{
A STROOCK FORMULA FOR A CERTAIN CLASS OF LÉVY PROCESSES AND APPLICATIONS TO FINANCE
}

\author{
M. EDDAHBI, J. L. SOLÉ, AND J. VIVES
}

Received 23 June 2004

We find a Stroock formula in the setting of generalized chaos expansion introduced by Nualart and Schoutens for a certain class of Lévy processes, using a Malliavin-type derivative based on the chaotic approach. As applications, we get the chaotic decomposition of the local time of a simple Lévy process as well as the chaotic expansion of the price of a financial asset and of the price of a European call option. We also study the behavior of the tracking error in the discrete delta neutral hedging under both the equivalent martingale measure and the historical probability.

\section{Introduction}

In general, a Lévy process has not the chaotic representation property (say CRP for brevity) but Nualart and Schoutens have developed in [8] a kind of generalized CRP for a large class of Lévy processes. This work enabled to define a Malliavin-type derivative using the chaotic approach in a recent paper of Léon et al. [6]. The main goal of the present article is to get a Stroock formula in this setting. This formula gives the kernels of the chaotic decomposition of smooth random variables as functionals of the underlying Lévy process. For a complete survey on Lévy processes, we refer to $[2,14]$.

We apply this formula to obtain the chaos decomposition of some functionals of simple Lévy processes. These processes are the sum of a Wiener process and $m$ independent Poisson processes. As it is pointed by the name, they are easy to handle and very useful for doing simulations since they approximate the square integrable compound Poisson process in the $L^{2}(\Omega \times[0, T])$ sense (see [6]). In particular, they approximate the process given by the sum of jumps with size greater than $\varepsilon>0$.

In this paper, firstly, we obtain the decomposition of the local time $L(t, x)$ defined as the density of the occupation measure. Secondly, taking account that these simple Lévy processes have been studied in [6] for pricing and hedging options in financial markets driven by such processes, we apply the Stroock formula to obtain the chaos expansion of an asset price as well as the price of a European call option based on this asset. The chaotic approach enable us to study the asymptotic behavior of the variance, since the terms of the chaotic expansion are orthogonal and in particular uncorrelated, and this is useful in practical hedging. 


\section{A Stroock formula for Lévy processes and applications}

The paper is organized as follows. The second section is devoted to recall some definitions and results related to Lévy chaotic calculus as well as to give some new remarks. In the third section, we get the Stroock formula. The fourth section is devoted to apply it in order to obtain the chaotic expansion of the local time of a simple Lévy process. Finally, the last three sections discuss chaotic expansions for the price of a financial asset and the price of a European call option, in a simple jump-diffusion model as well as the asymptotic behavior of the variance of the tracking error for discrete delta neutral hedging with respect to the mesh of the subdivision and under both the equivalent martingale measure and the historical probability.

\section{Basic elements of Lévy chaotic calculus}

2.1. The Teugels martingales family associated to a Lévy process. Let $X=\left\{X_{t}: t \geq 0\right\}$ be a real-valued Lévy process defined on a complete probability space $(\Omega, \mathscr{F}, \mathbb{P})$. Henceforth, we always assume that we are using the càdlàg version. Let $\left\{\mathscr{F}_{t}: t \geq 0\right\}$ be the natural filtration of $X$ completed with the $\mathbb{P}$-null sets of $\mathscr{F}$. We also assume that the Lévy measure $\nu$ of $X$ satisfies the following condition: there exist $\varepsilon>0$ and $\delta>0$ such that

$$
\int_{(-\varepsilon, \mathcal{\varepsilon})^{c}} e^{\delta|x|} \nu(d x)<+\infty
$$

where $(-\varepsilon, \varepsilon)^{c}$ stands for the complement of the interval $(-\varepsilon, \varepsilon)$. This implies that $X_{t}$ has moments of all orders and that the polynomials are dense in $L^{2}\left(\mathbb{R}, \mathbb{P} \circ X_{1}^{-1}\right)$ (see [8]).

Define

$$
X_{t}^{(1)}=X_{t}, \quad X_{t}^{(i)}=\sum_{0<s \leq t}\left(\Delta X_{s}\right)^{i}, \quad i \geq 2 .
$$

We have the following.

(i) The processes $X^{(i)}=\left\{X_{t}^{(i)}: t \geq 0\right\}, i=1,2, \ldots$, are Lévy processes that jump at the same points as $X$.

(ii) $\mathbb{E}\left[X_{t}^{(i)}\right]=m_{i} t$, where $m_{1}=\mathbb{E}\left[X_{1}\right]$ and $m_{i}=\int_{-\infty}^{\infty} x^{i} v(d x), i \geq 2$.

Now define

$$
Y_{t}^{(i)}=X_{t}^{(i)}-m_{i} t, \quad i=1,2, \ldots
$$

(iii) The processes $Y^{(i)}=\left\{Y_{t}^{(i)}: t \geq 0\right\}$ are martingales. The predictable quadratic covariation process of $Y^{(i)}$ and $Y^{(j)}$ is given by

$$
\left\langle Y^{(i)}, Y^{(j)}\right\rangle_{t}=m_{i+j} t, \quad i, j \geq 2 .
$$

Now, we introduce the so-called Teugels's martingales,

$$
H_{t}^{(i)}=\sum_{j=1}^{i} a_{i, j} Y_{t}^{(j)}, \quad i=1,2, \ldots,
$$

where the constants $a_{i, j}$ are chosen in such a way that $a_{i, 1}=1$ and the martingales $H^{(i)}, i=$ $1,2, \ldots$ are strongly orthogonal, that means, for $i \neq j$, the process $H^{(i)} H^{(j)}$ is a martingale. 
In particular, since $\left\langle H^{(i)}, H^{(j)}\right\rangle_{t}$ is a predictable process such that $H_{t}^{(i)} H_{t}^{(j)}-\left\langle H^{(i)}, H^{(j)}\right\rangle_{t}$ is a martingale, we have that

$$
\left\langle H^{(i)}, H^{(j)}\right\rangle_{t}=0, \quad i \neq j .
$$

Moreover, we have the following.

(iv) The processes $\left\{H_{t}^{(i)}: t \geq 0\right\}$ are martingales with predictable quadratic variation process given by

$$
\left\langle H^{(i)}, H^{(i)}\right\rangle_{t}=q_{i} t
$$

where

$$
q_{i}=\sum_{j, j^{\prime}=1, \ldots, i} a_{i, j} a_{i, j^{\prime}} m_{j+j^{\prime}}+a_{i, 1}^{2} \sigma^{2}
$$

2.2. Iterated integrals and a generalized CRP. Let $\Sigma_{n}=\left\{\left(t_{1}, \ldots, t_{n}\right) \in \mathbb{R}_{+}^{n}: 0<t_{1}<t_{2}<\right.$ $\left.\cdots<t_{n}\right\}$ be the positive simplex of $\mathbb{R}^{n}$. Given $f \in L^{2}\left(\Sigma_{n}\right)$, we will denote by $J_{n}^{\left(i_{1}, \ldots, i_{n}\right)}(f)$ the iterated integral of $f$ with respect to $H^{\left(i_{1}\right)}, \ldots, H^{\left(i_{n}\right)}$ :

$$
J_{n}^{\left(i_{1}, \ldots, i_{n}\right)}(f)=\int_{0}^{\infty}\left(\int_{0}^{t_{n}-} \cdots\left(\int_{0}^{t_{2}-} f\left(t_{1}, \ldots, t_{n}\right) d H^{\left(i_{1}\right)}\left(t_{1}\right)\right) \cdots d H^{\left(i_{n-1}\right)}\left(t_{n-1}\right)\right) d H^{\left(i_{n}\right)}\left(t_{n}\right) .
$$

We remark that all these integrals are well defined since all the processes $H^{(i)}$ for $i \geq 1$ are martingales with respect to the filtration $\left\{\mathscr{F}_{t}: t \geq 0\right\}$. Remark also that these iterated integrals are not the usual multiple stochastic integrals.

As a consequence of the strong orthogonality of the family of Teugels martingales, we have the following proposition.

Proposition 2.1. Let $f$ and $g$ belong to $L^{2}\left(\Sigma_{n}\right)$. Then

$$
\mathbb{E}\left[J_{n}^{\left(i_{1}, \ldots, i_{n}\right)}(f) J_{m}^{\left(j_{1}, \ldots, j_{m}\right)}(g)\right]=\left\{\begin{aligned}
q_{i_{1}} \cdots & q_{i_{n}} \int_{\Sigma_{n}} f\left(t_{1}, \ldots, t_{n}\right) g\left(t_{1}, \ldots, t_{n}\right) d t_{1} \cdots d t_{n} \\
& \text { if } n=m \text { and }\left(i_{1}, \ldots, i_{n}\right)=\left(j_{1}, \ldots, j_{n}\right), \\
0, & \text { otherwise. }
\end{aligned}\right.
$$

Finally, we recall the chaotic representation property of the square integrable random variables, one of the main results in [8] (see also [6]).

Proposition 2.2. Let $F \in L^{2}(\Omega, \mathscr{F}, \mathbb{P})$. Then $F$ has a unique representation of the form

$$
F=\mathbb{E}[F]+\sum_{n=1}^{\infty} \sum_{i_{1}, \ldots, i_{n}} J_{n}^{\left(i_{1}, \ldots, i_{n}\right)}\left(f_{i_{1}, \ldots, i_{n}}\right)
$$

where $f_{i_{1}, \ldots, i_{n}} \in L^{2}\left(\Sigma_{n}\right)$. 
214 A Stroock formula for Lévy processes and applications

\subsection{Derivative operators}

Definition 2.3. Let $f \in L^{2}\left(\Sigma_{n}\right)$. Set

$$
D_{t}^{(\ell)} J_{n}^{\left(i_{1}, \ldots, i_{n}\right)}(f)=\sum_{k=1}^{n} \mathbf{1}_{\left\{i_{k}=\ell\right\}} J_{n-1}^{\left(i_{1}, \ldots, \hat{i}_{k}, \ldots, i_{n}\right)}\left(f(\underbrace{\cdots}_{k-1}, t, \cdots) \mathbf{1}_{\Sigma_{n}^{(k)}(t)}(\cdot)\right),
$$

where

$$
\Sigma_{n}^{(k)}(t)=\left\{\left(t_{1}, \ldots, \hat{t}_{k}, \ldots, t_{n}\right) \in \Sigma_{n-1}: 0<t_{1}<\cdots<t_{k-1}<t \leq t_{k+1}<\cdots<t_{n}\right\}
$$

and $\hat{i}$ means that the $i$ th index is omitted.

Observe that if $k \neq k^{\prime}$, then $\Sigma_{n}^{(k)}(t) \cap \Sigma_{n}^{\left(k^{\prime}\right)}(t)=\varnothing$.

Now, we define the spaces of the random variables that are differentiable in the $\ell$ th direction. For this, we define the following subset of $L^{2}(\Omega)$ :

$$
\begin{aligned}
\mathbb{D}^{(\ell)_{1}, 2}=\{ & F \in L^{2}(\Omega): \sum_{n=1}^{\infty} \sum_{i_{1}, \ldots, i_{n}} \sum_{k=1}^{n} \mathbf{1}_{\left\{i_{k}=\ell\right\}} q_{i_{1}} \cdots \hat{q}_{i_{k}} \cdots q_{i_{n}} \\
& \left.\times \int_{0}^{\infty}\left\|f_{i_{1}, \ldots, i_{n}}(\cdot, t, \cdot) \mathbf{1}_{\Sigma_{n}^{(k)}(t)}\right\|_{L^{2}\left([0, \infty)^{n-1}\right)}^{2} d t<\infty\right\} .
\end{aligned}
$$

Definition 2.4. Given $F \in \mathbb{D}^{(\ell)_{1}, 2}$, we define the derivative of $F$ in the $\ell$ th direction as the element of $L^{2}(\Omega \times \mathbb{R})$ given by

$$
D_{t}^{(\ell)} F=\sum_{n=1}^{\infty} \sum_{i_{1}, \ldots, i_{n}} \sum_{k=1}^{n} \mathbf{1}_{\left\{i_{k}=\ell\right\}} J_{n-1}^{\left(i_{1}, \ldots, \hat{i}_{k}, \ldots, i_{n}\right)}\left(f_{i_{1}, \ldots, i_{n}}(\cdots, t, \cdots) \mathbf{1}_{\Sigma_{n}^{(k)}(t)}(\cdot)\right) .
$$

Observe that, as in the classical situation for Gaussian processes, $\mathbb{D}^{(\ell)_{1}, 2}$ is dense in $L^{2}(\Omega)$, since the elements of $L^{2}(\Omega)$ with a finite chaotic expansion are in $\mathbb{D}^{(\ell)_{1}, 2}$.

Set $\mathbb{D}^{(\infty)_{1}, 2}=\cap_{\ell \in \mathbb{N}} \mathbb{D}^{(\ell)_{1}, 2}$. Define also for $r>1$,

$$
\mathbb{D}^{(\ell)_{r}, 2}=\left\{F \in L^{2}(\Omega): D^{\left(\ell_{1}, \ldots, \ell_{r}\right)} F \in L^{2}\left([0, \infty)^{r} \times \Omega\right)\right\}
$$

and $\mathbb{D}^{\infty}=\cap_{r \in \mathbb{N}} \cap_{\ell \in \mathbb{N}^{r}} \mathbb{D}^{(\ell)_{r}, 2}$.

2.4. Simple Lévy processes. A simple Lévy process is given by

$$
X_{t}=\sigma W_{t}+\alpha_{1} N_{t}^{1}+\cdots+\alpha_{m} N_{t}^{m}, \quad t \geq 0
$$

where $\left\{W_{t}: t \geq 0\right\}$ is a standard Brownian motion, $\left\{N_{t}^{j}: t \geq 0\right\}, j=1, \ldots, m$, are independent Poisson processes of parameters $\lambda_{1}, \ldots, \lambda_{m}$, respectively, $\sigma>0$ and $\alpha_{1}, \ldots, \alpha_{m}$ are different nonnull numbers. The Lévy measure of $X$ is $\nu=\sum_{j=1}^{m} \lambda_{j} \delta_{\alpha_{j}}$ and satisfies the condition (2.1) of [8] for the validity of the chaotic representation property. 
Observe that

$$
\begin{aligned}
& X_{t}^{(1)}=X_{t}=\sigma W_{t}+\sum_{j=1}^{m} \alpha_{j} N_{t}^{j}, \\
& X_{t}^{(i)}=\sum_{0<s \leq t}\left(\Delta X_{s}\right)^{i}=\sum_{j=1}^{m} \alpha_{j}^{i} N_{t}^{j}, \quad i \geq 2,
\end{aligned}
$$

because the independence of two Poisson processes adapted to the same filtration implies that they do not jump at the same time (see [2]).

Since

$$
Y_{t}^{(i)}=X_{t}^{(i)}-m_{i} t, \quad i \geq 1
$$

we have

$$
\begin{aligned}
& Y_{t}^{(1)}=\sigma W_{t}+\sum_{j=1}^{m} \alpha_{j}\left(N_{t}^{j}-\lambda_{j} t\right), \\
& Y_{t}^{(i)}=\sum_{j=1}^{m} \alpha_{j}^{i}\left(N_{t}^{j}-\lambda_{j} t\right), \quad i \geq 2 .
\end{aligned}
$$

Then, the martingales $Y^{(i)}, i \geq 1$, are linear combinations of $W_{t}, N_{t}^{1}-\lambda_{1} t, \ldots, N_{t}^{m}-\lambda_{m} t$. Since the martingales $H^{(i)}$ are linear combinations of $Y^{(1)}, \ldots, Y^{(i)}$, it follows that $H^{(i)}$ are also linear combinations of $W_{t}, \tilde{N}_{t}^{1}, \ldots, \tilde{N}_{t}^{m}$, where $\tilde{N}_{t}^{j}=N_{t}^{j}-\lambda_{j} t$. Therefore, we have a CRP in terms of the iterated integrals with respect to $W_{t}, \tilde{N}_{t}^{1}, \ldots, \tilde{N}_{t}^{m}$. Recall also that by [6, Proposition 1.10], $H^{(i)}=0$, for $i \geq m+2$. Furthermore, since we are assuming that $\alpha_{1}, \ldots, \alpha_{m}$ are different, there is one and only one way to express $Y^{(1)}, \ldots, Y^{(m+1)}$ as linear combinations of $W_{t}, \tilde{N}_{t}^{1}, \ldots, \tilde{N}_{t}^{m}$ since the uniqueness of the CRP in terms of the $W_{t}, \tilde{N}_{t}^{1}, \ldots, \tilde{N}_{t}^{m}$.

To unify the notations, we will write $G_{0}(t)=W_{t}$ and $G_{j}(t)=\tilde{N}_{t}^{j}$ for $j=1, \ldots, m$. Also, we will denote by $L_{n}^{\left(i_{1}, \ldots, i_{n}\right)}(f)$ the iterated integral of $f$ with respect to $G_{i_{1}}, \ldots, G_{i_{n}}$ :

$$
L_{n}^{\left(i_{1}, \ldots, i_{n}\right)}(f)=\int_{0}^{\infty} \int_{0}^{t_{n}^{-}} \cdots \int_{0}^{t_{2}^{-}} f\left(t_{1}, \ldots, t_{n}\right) d G_{i_{1}}\left(t_{1}\right) \cdots d G_{i_{n-1}}\left(t_{n-1}\right) d G_{i_{n}}\left(t_{n}\right) .
$$

Thus, we have also the chaotic representation property in terms of the $G_{i}$ 's.

Proposition 2.5. Let $F \in L^{2}(\Omega, \mathscr{F}, \mathbb{P})$. Then $F$ has a representation of the form

$$
F=\mathbb{E}[F]+\sum_{n=1}^{\infty} \sum_{0 \leq i_{1}, \ldots, i_{n} \leq m} L_{n}^{\left(i_{1}, \ldots, i_{n}\right)}\left(f_{i_{1}, \ldots, i_{n}}\right),
$$

where $f_{i_{1}, \ldots, i_{n}} \in L^{2}\left(\Sigma_{n}\right)$.

In this setting, we recall a result of Léon et al. [6] which says that is possible to compute the derivatives in the directions $W, N^{1}, \ldots, N^{m}, \ldots$, following the classical rules on each space. Recall that in Poisson setting the derivative is a difference operator (see [10]). 
We use the space $\Omega=\Omega_{0} \times \Omega_{1} \times \cdots \times \Omega_{m}$, where $\Omega_{0}$ is the canonical space of the Wiener process and $\Omega_{i}, i=1, \ldots, m$, are, respectively, the canonical spaces of Poisson processes $N^{i}, i=1, \ldots, m$, that are the spaces of all possible paths of Poisson's.

Remark 2.6. If we iterate the derivative with respect to the Poisson's $i$ and $j(i<j)$, we obtain

$$
\begin{aligned}
D_{t_{2}}^{i} D_{t_{1}}^{j} F= & F\left(\omega_{0}, \ldots, \omega_{i}+\delta_{t_{2}}, \ldots, \omega_{j}+\delta_{t_{1}}, \ldots, \omega_{m}\right) \\
& -F\left(\omega_{0}, \ldots, \omega_{i}, \ldots, \omega_{j}+\delta_{t_{1}}, \ldots, \omega_{m}\right) \\
& -F\left(\omega_{0}, \ldots, \omega_{i}+\delta_{t_{2}}, \ldots, \omega_{j}, \ldots, \omega_{m}\right)+F\left(\omega_{0}, \ldots, \omega_{m}\right) .
\end{aligned}
$$

Observe that $D_{t_{2}}^{i} D_{t_{1}}^{j} F=D_{t_{1}}^{j} D_{t_{2}}^{i} F$, but this equality is not true in general if we only interchange the superindexes or the subindexes. If the iteration is done with respect to the same direction $i \geq 1$, we obtain that $D_{t_{1}, \ldots, t_{s}}^{i, \ldots, i} F$, denoted by $D_{J_{s}}^{i, s} F$ where $J_{s}=\left\{t_{1}, \ldots, t_{s}\right\}$, is equal to $\sum_{B \subset J_{s}}(-1)^{s-(\# B)} F\left(\omega_{0}, \ldots, \omega_{i}+\sum_{t_{j} \in B} \delta_{t_{j}}, \ldots, \omega_{m}\right)$ where we have used the convention $\sum_{t_{j} \in \varnothing} \delta_{t_{j}}=0$.

Remark 2.7. It is clear that $D_{t_{1}}^{0} D_{t_{2}}^{i} F=D_{t_{2}}^{i} D_{t_{1}}^{0} F$ for all $i \geq 1$. We want to compute $D_{t_{1}, \ldots, t_{n}}^{i_{1}, \ldots, i_{n}} F$ for $i_{1}, \ldots, i_{n} \in\{0,1, \ldots, m\}$. To do this, we define $I_{s}=\left\{j, i_{j}=s\right\}$ and $J_{s}=\left\{t_{j}, j \in I_{s}\right\}$ for $s \in\{0,1, \ldots, m\}$. Then $k_{s}=\# I_{s}$ will be the order of derivation with respect to $G_{s}$, and hence $\sum_{i=0}^{m} k_{i}=n$ and $\cup_{i=0}^{m} J_{i}=\left\{t_{1}, \ldots, t_{n}\right\}$. Therefore,

$$
D_{t_{1}, \ldots, t_{n}}^{i_{1}, \ldots, i_{n}} F=D_{J_{0}}^{0, k_{0}} D_{J_{1}}^{1, k_{1}} \cdots D_{J_{m}}^{m, k_{m}} F
$$

with the convention that $D_{J_{i}}^{i, 0}$ is the identity, and we get

$$
D_{t_{1}, \ldots, t_{n}}^{i_{1}, \ldots, i_{n}} F=D_{J_{0}}^{0, k_{0}}\left[\sum_{B_{i} \subset J_{i}, 1 \leq i \leq m}(-1)^{\sum_{i=1}^{m}\left(k_{i}-\left(\# B_{i}\right)\right)} F\left(\omega_{0}, \omega_{1}+\sum_{t_{j} \in B_{1}} \delta_{t_{j}}, \ldots, \omega_{m}+\sum_{t_{j} \in B_{m}} \delta_{t_{j}}\right)\right] .
$$

Remark 2.8. If $F=f\left(W_{t}, \tilde{N}_{t}^{1}, \ldots, \tilde{N}_{t}^{m}\right)$, where $f$ is a smooth function, its derivative $D_{s}^{i} F$ for $i \geq 1$ and $s \leq t$ is the difference $f\left(W_{t}, \tilde{N}_{t}^{1}, \ldots, \tilde{N}_{t}^{i}+1, \ldots, \tilde{N}_{t}^{m}\right)-f\left(W_{t}, \tilde{N}_{t}^{1}, \ldots, \tilde{N}_{t}^{m}\right)$. So in general

$$
\begin{aligned}
D_{t_{1}, \ldots, t_{n}}^{i_{1}, \ldots, i_{n}} F= & D_{J_{0}}^{0, k_{0}} D_{J_{1}}^{1, k_{1}} \cdots D_{J_{m}}^{m, k_{m}} F \\
= & D_{J_{0}}^{0, k_{0}}\left[\sum_{0 \leq j_{i} \leq k_{i}, 1 \leq i \leq m}(-1)^{n-k_{0}-\sum_{i=1}^{m} j_{i}}\right. \\
& \left.\quad \times \prod_{i=1}^{m}\left(\begin{array}{c}
k_{i} \\
j_{i}
\end{array}\right) f\left(W_{t}, \tilde{N}_{t}^{1}+j_{1}, \ldots, \tilde{N}_{t}^{m}+j_{m}\right)\right] \prod_{i=1}^{m} \mathbf{1}_{\left\{J_{i} \subset[0, t]^{\left.k_{i}\right\}}\right.} \\
= & \sum_{0 \leq j_{i} \leq k_{i}, 1 \leq i \leq m}(-1)^{n-k_{0}-\sum_{i=1}^{m} j_{i}} \\
& \quad \times \prod_{i=1}^{m}\left(\begin{array}{c}
k_{i} \\
j_{i}
\end{array}\right) \frac{\partial^{k_{0}} f}{\partial x_{1}^{k_{0}}}\left(W_{t}, \tilde{N}_{t}^{1}+j_{1}, \ldots, \tilde{N}_{t}^{m}+j_{m}\right) \prod_{i=0}^{m} \mathbf{1}_{\left\{J_{i} \subset[0, t]^{k_{i}}\right\}} .
\end{aligned}
$$


Remark 2.9. If there's only one Poisson process and no Brownian part, different authors as Léon et al. [7] and Privault [12] have used the iterated derivatives to find the chaotic decomposition of functionals of the $d$ first jump times of the process. In the present article, we consider different Poisson processes and a Brownian part. Moreover, we are not restricted to a specified finite number of jump times.

\section{Stroock formula for Lévy processes}

The aim of this section is to derive a Stroock formula for functionals of a Lévy process belonging to $\mathbb{D}^{\infty}$. First we deal with the case that $F$ is an element of a specific chaos and finally we will extend the result to $F$ in $\mathbb{D}^{\infty}$.

We start with the following lemma.

LEMMA 3.1. If $F=J_{n}^{i_{1}, \ldots, i_{n}}(f)$, then

$$
D_{t_{1}, \ldots, t_{n}}^{j_{1}, \ldots, j_{n}} F=\sum_{\sigma \in \mathscr{P}\{1, \ldots, n\}} \delta_{j_{\sigma(1), i_{1}}} \cdots \delta_{j_{\sigma(n), i_{n}}} f\left(t_{\sigma(1)}, \ldots, t_{\sigma(n)}\right) \mathbf{1}_{\left\{t_{\sigma(1)}<\cdots<t_{\sigma(n)}\right\}},
$$

where $\mathscr{P}\{1, \ldots, n\}$ is the set of permutations of $\{1, \ldots, n\}$.

Proof. First remark that, in the sum, only one term is different from zero. To prove the lemma, we use induction on $n$.

For $n=2$, we have

$$
\begin{aligned}
& D_{t_{1}}^{j_{1}}\left(\int_{0}^{1} \int_{0}^{r_{2}-} f\left(r_{1}, r_{2}\right) d H^{\left(i_{1}\right)}\left(r_{1}\right) d H^{\left(i_{2}\right)}\left(r_{2}\right)\right) \\
& \quad=\delta_{j_{1}, i_{1}} \int_{t_{1}}^{1} f\left(t_{1}, r_{2}\right) d H^{\left(i_{2}\right)}\left(r_{2}\right)+\delta_{j_{1}, i_{2}} \int_{0}^{t_{1}} f\left(r_{1}, t_{1}\right) d H^{\left(i_{1}\right)}\left(r_{1}\right) \\
& \quad=\delta_{j_{1}, i_{1}} \int_{0}^{1} f\left(t_{1}, r_{2}\right) \mathbf{1}_{\left[t_{1}, 1\right]}\left(r_{2}\right) d H^{\left(i_{2}\right)}\left(r_{2}\right)+\delta_{j_{1}, i_{2}} \int_{0}^{1} f\left(r_{1}, t_{1}\right) \mathbf{1}_{\left[0, t_{1}\right]}\left(r_{1}\right) d H^{\left(i_{1}\right)}\left(r_{1}\right),
\end{aligned}
$$

and applying now the operator $D_{t_{2}}^{j_{2}}$, we get

$$
D_{t_{2}}^{j_{2}} D_{t_{1}}^{j_{1}}\left(J_{2}^{i_{1}, i_{2}}(f)\right)=\delta_{j_{2}, i_{2}} \delta_{j_{1}, i_{1}} f\left(t_{1}, t_{2}\right) \mathbf{1}_{\left[t_{1}, 1\right]}\left(t_{2}\right)+\delta_{j_{2}, i_{1}} \delta_{j_{1}, i_{2}} f\left(t_{2}, t_{1}\right) \mathbf{1}_{\left[0, t_{1}\right]}\left(t_{2}\right) .
$$

Hence, if $t_{1}<t_{2}$, we have

$$
D_{t_{2}}^{j_{2}} D_{t_{1}}^{j_{1}}\left(\int_{0}^{1} \int_{0}^{r_{2}-} f\left(r_{1}, r_{2}\right) d H^{\left(i_{1}\right)}\left(r_{1}\right) d H^{\left(i_{2}\right)}\left(r_{2}\right)\right)=\delta_{j_{1}, i_{1}} \delta_{j_{2}, i_{2}} f\left(t_{1}, t_{2}\right) .
$$

Therefore, the formula (3.1) is satisfied for $n=2$.

Besides,

$$
\begin{aligned}
D_{t_{1}, \ldots, t_{n}}^{j_{1}, \ldots, j_{n}}\left(J_{n}^{i_{1}, \ldots, i_{n}}(f)\right) & =D_{t_{j_{n}}}^{j_{n}}\left(\cdots\left(D_{t_{j_{1}}}^{j_{1}}\left(J_{n}^{i_{1}, \ldots, i_{n}}(f)\right)\right)\right) \\
& =D_{t_{j_{n}}}^{j_{n}} \cdots D_{t_{j_{2}}}^{j_{2}}\left[\sum_{k=1}^{n} \delta_{i_{k} j_{1}} J_{n-1}^{i_{1}, \ldots, \hat{i}_{k}, \ldots, i_{n}}\left(f\left(\ldots, t_{1}, \ldots,\right) \mathbf{1}_{\sum_{n}^{(k)}\left(t_{1}\right)}(\cdot)\right)\right] .
\end{aligned}
$$


218 A Stroock formula for Lévy processes and applications

and the induction hypothesis yields

$$
\begin{aligned}
D_{t_{1}, \ldots, t_{n}}^{j_{1}, \ldots, j_{n}} & \left(J_{n}^{i_{1}, \ldots, i_{n}}(f)\right) \\
= & \sum_{k=1}^{n} \delta_{i_{k} j_{1}} \sum_{\tau \in \mathscr{P}\left\{1, \ldots, \hat{k}_{k} \ldots, n\right\}}^{\infty} \delta_{j_{\tau(2), i_{1}}} \cdots \delta_{j_{\tau(k-1), i_{k-1}}} \delta_{j_{\tau(k+1), i_{k+1}}} \cdots \delta_{j_{\tau(n), i_{n}}} \\
& \times f\left(t_{\tau(2)}, \ldots, t_{\tau(k-1)}, t_{1}, t_{\tau(k+1)}, \ldots, t_{\tau(n)}\right) \mathbf{1}_{\left\{t_{\tau(2)}<\cdots<t_{\tau(k-1)}<t_{1}<t_{\tau(k+1)} \cdots<t_{\tau(n)}\right\}} \\
= & \sum_{\sigma \in \mathscr{P}\{1, \ldots, n\}} \delta_{j_{\sigma(1), i_{1}}} \cdots \delta_{j_{\sigma(n), i_{n}}} f\left(t_{\sigma(1)}, \ldots, t_{\sigma(n)}\right) \mathbf{1}_{\left\{t_{\sigma(1)}<\cdots<t_{\sigma(n)}\right\}} .
\end{aligned}
$$

Remark 3.2. If $t_{1}<\cdots<t_{n}$, we get the following equality:

$$
D_{t_{1}, \ldots, t_{n}}^{j_{1}, \ldots, j_{n}}\left(J_{n}^{i_{1}, \ldots, i_{n}}(f)\right)=\delta_{j_{1}, i_{1}} \cdots \delta_{j_{n}, i_{n}} f\left(t_{1}, \ldots, t_{n}\right) .
$$

Now, we apply Lemma 3.1 to obtain the Stroock formula.

Theorem 3.3. Let $F$ be in $\mathbb{D}^{\infty}$ with chaotic expansion

$$
F=\mathbb{E}[F]+\sum_{n=1}^{\infty} \sum_{0 \leq i_{1}, \ldots, i_{n} \leq m} J_{n}^{i_{1}, \ldots, i_{n}}\left(f_{i_{1}, \ldots, i_{n}}\right) .
$$

Then

$$
f_{i_{1}, \ldots, i_{n}}\left(t_{1}, \ldots, t_{n}\right)=\mathbb{E}\left[D_{t_{1}, \ldots, t_{n}}^{i_{1}, \ldots, i_{n}} F\right]
$$

where $t_{1}<\cdots<t_{n}$.

Proof. As the iterated derivatives of order $n$ of elements belonging to chaos of order less than $n$ are zero and applying the above lemma, we get

$$
D_{t_{1}, \ldots, t_{n}}^{i_{1}, \ldots, i_{n}} F=f_{i_{1}, \ldots, i_{n}}\left(t_{1}, \ldots, t_{n}\right)+M_{n}, \quad \text { with } t_{1}<\cdots<t_{n}
$$

where $M_{n}$ is a sum of variables in chaos of order greater than or equal to $n$. Taking the expectation, we obtain the desired formula.

\section{Chaos expansion of the local time of a simple Lévy process}

The aim of this section is to apply the Stroock formula to find the chaotic decomposition of the local time of a simple Lévy process.

We denote by $H_{n}$ the $n$th Hermite polynomial defined by

$$
H_{n}(x)=\frac{(-1)^{n}}{\sqrt{n !}} \exp \left(\frac{x^{2}}{2}\right) \frac{d^{n}}{d x^{n}}\left(\exp \left(-\frac{x^{2}}{2}\right)\right)
$$

for $n \geq 1$ and $H_{0}(x)=1$. 
We define the local time $L(t, x)$ of a Lévy process $X$ as the density of the occupation measure

$$
m_{t}(A)=\int_{0}^{t} \mathbf{1}_{A}\left(X_{s}\right) d s, \quad A \in \mathscr{B}(\mathbb{R}) .
$$

It is well known that this density $L(t, x)$ exists and is a nondecreasing function of $t$ and the measure $L(d t, x)$ is concentrated on the level set $\left\{s: X_{s}=x\right\}$. Moreover, Barlow [1] shows that $L(t, x)$ has an almost surely jointly continuous version.

It is well known also that we can write

$$
L(t, x)=\int_{0}^{t} \delta_{x}\left(X_{s}\right) d s
$$

To apply the Stroock formula, we consider

$$
L_{\varepsilon}(t, x)=\int_{0}^{t} p_{\varepsilon}\left(X_{s}-x\right) d s,
$$

where $p_{\varepsilon}$ is the centered Gaussian kernel with variance $\varepsilon>0$. The classical idea of approximating the Dirac distribution $\delta_{x}$ by $p_{\varepsilon}$ has been used to calculate the chaotic decomposition of the local time in the case of the Brownian motion by Nualart and Vives [11] and for the fractional Brownian motion by Coutin et al. [3] and Eddahbi et al. [4].

Before stating precise results of this section, we prove some technical lemmas.

LeMmA 4.1. Let $F=p_{\varepsilon}\left(\sigma W_{s}+\sum_{i=1}^{m} \alpha_{i} \tilde{N}_{s}^{i}-x\right)$. Then

$$
\begin{aligned}
& D_{t_{1}, \ldots, t_{n}}^{i_{1}, \ldots, i_{n}} F \\
& \quad=\sum_{i=1}^{m} \sum_{\ell_{i}=0}^{k_{i}} \prod_{j=1}^{m}\left(\begin{array}{c}
k_{j} \\
\ell_{j}
\end{array}\right)(-1)^{n-k_{0}-\sum_{j=1}^{m} \ell_{j}} \sigma^{k_{0}} p_{\varepsilon}^{\left(k_{0}\right)}\left(\sigma W_{s}+\sum_{i=1}^{m} \alpha_{i} \tilde{N}_{s}^{i}+\alpha_{i} \ell_{i}-x\right) \prod_{r=1}^{n} \mathbf{1}_{[0, s]}\left(t_{r}\right),
\end{aligned}
$$

where $k_{i}=\#\left\{j: i_{j}=i\right\}, i=0,1, \ldots, m$.

Proof. We only apply Remark 2.8 of Section 2.

Lemma 4.2. $\operatorname{Set} g_{n}(y)=H_{n}(y) \exp \left(-y^{2} / 2\right)$. Then

$$
\mathbb{E}\left[p_{\varepsilon}^{(n)}\left(\sigma W_{s}-a\right)\right]=\frac{(-1)^{n}}{\left(s \sigma^{2}+\varepsilon\right)^{(n+1) / 2}} \frac{\sqrt{n !}}{\sqrt{2 \pi}} g_{n}\left(\frac{a}{\sqrt{s \sigma^{2}+\varepsilon}}\right) .
$$

Proof. As $p_{\varepsilon}^{(n)}(x)=\left(1 / \sigma^{n+1}\right) p_{\varepsilon / \sigma^{2}}^{(n)}(x / \sigma)$, we have

$$
\mathbb{E}\left[p_{\varepsilon}^{(n)}\left(\sigma W_{s}-a\right)\right]=\frac{1}{\sigma^{n+1}} \mathbb{E}\left[p_{\varepsilon / \sigma^{2}}^{(n)}\left(W_{s}-\frac{a}{\sigma}\right)\right] .
$$

Interchanging the derivative operator and the expectation, and using

$$
p_{\varepsilon}^{(n)}(x)=(-1)^{n} \sqrt{n !} \frac{p_{\varepsilon}(x)}{\varepsilon^{n / 2}} H_{n}\left(\frac{x}{\sqrt{\varepsilon}}\right), \quad \mathbb{E}\left[p_{\varepsilon}\left(W_{s}-a\right)\right]=p_{s+\varepsilon}(a),
$$

we get the formula of the lemma. 
LEMma 4.3. For all $s>0$ and for all $n \geq 0$,

$$
\left|\mathbb{E}\left[p_{\varepsilon}^{(n)}\left(\sigma W_{s}-a\right)\right]\right| \leq \frac{1}{2 \pi} \Gamma\left(\frac{n+1}{2}\right)\left(\frac{s \sigma^{2}}{2}\right)^{-(n+1) / 2} .
$$

Proof. We apply Lemma 4.2 and the inequality $\left|g_{n}(y)\right| \leq\left(\left(2^{n / 2}\right) / \sqrt{\pi n !}\right) \Gamma((n+1) / 2)$ (see [4]).

Then, we can prove the following proposition.

Proposition 4.4. The chaos expansion of $L_{\varepsilon}(t, x)=\int_{0}^{t} p_{\varepsilon}\left(\sigma W_{s}+\sum_{i=1}^{m} \alpha_{i} \tilde{N}_{s}^{i}-x\right) d s$ is

$$
\begin{aligned}
L_{\varepsilon}(t, x)=\sum_{n=0}^{\infty} \sum_{0 \leq i_{1}, \ldots, i_{n} \leq m} L_{n}^{i_{1}, \ldots, i_{n}} & {\left[\sum_{i=1}^{m} \sum_{\ell_{i}=0}^{k_{i}} \prod_{j=1}^{m}\left(\begin{array}{c}
k_{j} \\
\ell_{j}
\end{array}\right) \sigma^{k_{0}}(-1)^{n-k_{0}-\sum_{j=1}^{m} \ell_{j}} \frac{\sqrt{k_{0} !}}{\sqrt{2 \pi}}\right.} \\
& \times \sum_{r_{1}=0}^{\infty} \cdots \sum_{r_{m}=0}^{\infty} \int_{t_{1} \vee \cdots \vee t_{n}}^{t} \frac{e^{-\lambda s}}{\left(s \sigma^{2}+\varepsilon\right)^{\left(k_{0}+1\right) / 2}} \\
& \left.\times \prod_{j=1}^{m} \frac{\left(\lambda_{j} s\right)^{r_{j}}}{r_{j} !} g_{n}\left(\frac{x-\sum_{j=1}^{m} \alpha_{j}\left(r_{j}-\lambda_{j} s+\ell_{j}\right)}{\sqrt{s \sigma^{2}+\varepsilon}}\right) d s\right],
\end{aligned}
$$

where $\lambda=\sum_{i=1}^{m} \lambda_{i}$.

Proof. We apply the Stroock formula to $p_{\varepsilon}\left(\sigma W_{s}+\sum_{i=1}^{m} \alpha_{i} \tilde{N}_{s}^{i}-x\right)$ and use Lemma 4.1 for the expression of the iterated derivative and Lemma 4.2 for the expectation with respect to the Wiener part. Besides, we calculate the expectation with respect to the Poisson parts.

Remark 4.5. Note that the kernels depend only on $k_{0}, k_{1}, \ldots, k_{m}$. Hence, it is the same for all sets of indexes $i_{1}, \ldots, i_{n}$ that derive to equal $k_{0}, k_{1}, \ldots, k_{m}$. Observe also that although the kernel is the same, the iterated integral $L_{n}^{i_{1}, \ldots, i_{n}}$ can depend on the order of the indexes as we will see in the next example:

$$
\int_{0}^{1} \int_{0}^{u_{2}-} d W_{u_{1}} d N_{u_{2}}=\int_{0}^{1} W_{u_{2}-} d N_{u_{2}}=\sum_{i=1}^{N_{1}} W_{T_{i}},
$$

where $T_{i}, i \in \mathbb{N}$, are the jump times of Poisson. But

$$
\begin{aligned}
\int_{0}^{1} \int_{0}^{u_{2}-} & d N_{u_{1}} d W_{u_{2}} \\
& =\int_{0}^{1} N_{u_{2}-} d W_{u_{2}} \\
& =\left[N_{1-}\left(W_{1}-W_{T_{N_{1}}}\right)+\left(N_{1-}-1\right)\left(W_{T_{N_{1}}}-W_{T_{N_{1}-1}}\right)+\cdots+\left(W_{T_{2}}-W_{T_{1}}\right)\right] \\
& =W_{1} N_{1-}-\sum_{i=1}^{N_{1}} W_{T_{i}} .
\end{aligned}
$$


In order to establish the chaotic expansion of the local time of a simple Lévy process we state the following lemma (see [11]).

LEMmA 4.6. Let $\left\{F_{\varepsilon}\right\}_{\varepsilon>0}$ be a family of square integrable random variables with the expansions

$$
F_{\varepsilon}=\mathbb{E}\left[F_{\varepsilon}\right]+\sum_{n=1}^{\infty} \sum_{i_{1}, \ldots, i_{n}} J_{n}^{\left(i_{1}, \ldots, i_{n}\right)}\left(f_{i_{1}, \ldots, i_{n}}^{\varepsilon}\right),
$$

where $f_{i_{1}, \ldots, i_{n}}^{\varepsilon}$ belongs to $L^{2}\left([0, \infty)^{n}\right)$.

Assume that

(i) $f_{i_{1}, \ldots, i_{n}}^{\varepsilon}$ converges in $L^{2}\left([0, \infty)^{n}\right)$, when $\varepsilon$ goes to zero, to some symmetric function $f_{i_{1}, \ldots, i_{n}} \in L^{2}\left([0, \infty)^{n}\right)$

(ii) $\sum_{n=1}^{\infty} \sum_{i_{1}, \ldots, i_{n}} q_{i_{1}} \cdots q_{i_{n}} \sup _{\varepsilon}\left\|f_{i_{1}, \ldots, i_{n}}^{\varepsilon}(\cdot)\right\|_{L^{2}\left([0, \infty)^{n}\right)}^{2}$ is convergent.

Then the family $F_{\varepsilon}$ converges in $L^{2}(\Omega)$ to $F=\mathbb{E}[F]+\sum_{n=1}^{\infty} \sum_{i_{1}, \ldots, i_{n}} J_{n}^{\left(i_{1}, \ldots, i_{n}\right)}\left(f_{i_{1}, \ldots, i_{n}}\right)$.

Then we can prove the following proposition.

Proposition 4.7. For each $x \in \mathbb{R}$ and $t \in[0, T]$, the random variables

$$
\int_{0}^{t} p_{\varepsilon}\left(\sigma W_{s}+\sum_{i=1}^{m} \alpha_{i} \tilde{N}_{s}^{i}-x\right) d s
$$

converge to $L(t, x)$ in $L^{2}(\Omega)$ as $\varepsilon$ tends to zero. Furthermore, with $\lambda=\sum_{i=1}^{m} \lambda_{i}$, the local time $L(t, x)$ has the following chaotic decomposition:

$$
\begin{aligned}
& L(t, x)=\sum_{n=0}^{\infty} \sum_{0 \leq i_{1}, \ldots, i_{n} \leq m} L_{n}^{i_{1}, \ldots, i_{n}}\left[\sum_{i=1}^{m} \sum_{\ell_{i}=0}^{k_{i}}\left(\prod_{j=1}^{m}\left(\begin{array}{l}
k_{j} \\
\ell_{j}
\end{array}\right)\right) \sigma^{k_{0}}\right. \\
& \times(-1)^{n-k_{0}-\sum_{j=1}^{m} \ell_{j}} \frac{\sqrt{k_{0} !}}{\sqrt{2 \pi}} \sum_{r_{1}=0}^{\infty} \cdots \sum_{r_{m}=0}^{\infty} \int_{t_{1} \vee \cdots \vee t_{n}}^{t} \frac{e^{-\lambda s}}{\left(s \sigma^{2}\right)^{\left(k_{0}+1\right) / 2}} \\
&\left.\times \prod_{j=1}^{m} \frac{\left(\lambda_{j} s\right)^{r_{j}}}{r_{j} !} g_{n}\left(\frac{x-\sum_{j=1}^{m} \alpha_{j}\left(r_{j}-\lambda_{j} s+\ell_{j}\right)}{\sigma \sqrt{s}}\right) d s\right]
\end{aligned}
$$

Remark 4.8. Note that Lemma 4.6, simultaneously, proves that the local time is in $L^{2}(\Omega)$ and gives its chaotic expansion.

Proof. We must check the two hypotheses of Lemma 4.6. We start with (ii). By Lemma 4.1, we have

$$
\begin{aligned}
\left|\mathbb{E}\left[D_{t_{1}, \ldots, t_{n}}^{i_{1}, \ldots, i_{n}} L_{\varepsilon}(t, x)\right]\right| & \\
\leq & \sum_{i=1}^{m} \sum_{\ell_{i}=0}^{k_{i}} \prod_{j=1}^{m}\left(\begin{array}{c}
k_{j} \\
\ell_{j}
\end{array}\right) \sum_{r_{1}=0}^{\infty} \cdots \sum_{r_{m}=0}^{\infty} \int_{t_{1} \vee \cdots \vee t_{n}}^{t} e^{-\lambda s} \prod_{j=1}^{m} \frac{\left(\lambda_{j} s\right)^{r_{j}}}{r_{j} !} \sigma^{k_{0}} \\
& \times \mathbb{E}_{W}\left[p_{\varepsilon}^{\left(k_{0}\right)}\left(\sigma W_{s}+\sum_{j=1}^{m} \alpha_{j}\left(r_{j}-\lambda_{j} s+\ell_{j}\right)-x\right)\right] \prod_{r=1}^{n} \mathbf{1}_{[0, s]}\left(t_{r}\right) d s,
\end{aligned}
$$


222 A Stroock formula for Lévy processes and applications

and by Lemma 4.3, and since

$$
e^{-\lambda s} \prod_{j=1}^{m} \sum_{r_{j}=0}^{\infty} \frac{\left(\lambda_{j} s\right)^{r_{j}}}{r_{j} !}=1
$$

the right-hand side of (4.16) is bounded by

$$
\begin{gathered}
\sum_{i=1}^{m} \sum_{\ell_{i}=0}^{k_{i}} \frac{\sigma^{k_{0}}}{2 \pi} \prod_{j=1}^{m}\left(\begin{array}{c}
k_{j} \\
\ell_{j}
\end{array}\right) \int_{t_{1} \vee \cdots \vee t_{n}}^{t} \frac{\Gamma\left(\left(k_{0}+1\right) / 2\right)}{\left(\left(s \sigma^{2}\right) / 2\right)^{\left(k_{0}+1\right) / 2}} d s \\
\quad=\frac{2^{n-\left(k_{0} / 2\right)+(1 / 2)}}{2 \pi \sigma} \Gamma\left(\frac{k_{0}+1}{2}\right) \int_{t_{1} \vee \cdots \vee t_{n}}^{t} \frac{d s}{s^{\left(k_{0}+1\right) / 2}},
\end{gathered}
$$

where we have used the identity

$$
\sum_{i=1}^{m} \sum_{\ell_{i}=0}^{k_{i}} \prod_{j=1}^{m}\left(\begin{array}{l}
k_{j} \\
\ell_{j}
\end{array}\right)=2^{n-k_{0}}
$$

Therefore,

$$
\left|\mathbb{E}\left[D_{t_{1}, \ldots, t_{n}}^{i_{1}, \ldots, i_{n}} L_{\varepsilon}(t, x)\right]\right|^{2} \leq \frac{2^{2 n-k_{0}+1}}{(2 \pi \sigma)^{2}} \Gamma\left(\frac{k_{0}+1}{2}\right)^{2}\left(\int_{t_{1} \vee \cdots \vee t_{n}}^{t} \frac{d s}{s^{\left(k_{0}+1\right) / 2}}\right)^{2} .
$$

Then

$$
\begin{aligned}
\left\|\mathbb{E}\left[D_{t_{1}, \ldots, t_{n}}^{i_{1}, \ldots, i_{n}} L_{\varepsilon}(t, x)\right]\right\|_{L^{2}\left([0, T]^{n}\right)}^{2} \\
\quad \leq \frac{2^{2 n-k_{0}+1}}{(2 \pi \sigma)^{2}} \Gamma\left(\frac{k_{0}+1}{2}\right)^{2} \int_{0}^{t} \frac{y^{n-1} d y}{(n-1) !}\left(\int_{y}^{t} s^{\left(k_{0}+1\right) / 2} d s\right)^{2} \\
=\frac{2^{2 n-k_{0}+1}}{(2 \pi \sigma)^{2}} \Gamma\left(\frac{k_{0}+1}{2}\right)^{2} \int_{0}^{t} \int_{0}^{t} \int_{0}^{t} \frac{y^{n-1} \mathbf{1}_{[0, u \wedge v]}(y) d y}{(n-1) !(u v)^{\left(k_{0}+1\right) / 2}} d u d v \\
=\frac{2^{2 n-k_{0}}}{n !(\pi \sigma)^{2}} \Gamma\left(\frac{k_{0}+1}{2}\right)^{2} \int_{0}^{t} v^{-\left(k_{0}+1\right) / 2} d v \int_{0}^{v} u^{n-\left(\left(k_{0}+1\right) / 2\right)} d u \\
=\frac{2^{2 n-k_{0}}}{n !(\pi \sigma)^{2}} \Gamma\left(\frac{k_{0}+1}{2}\right)^{2} \frac{2}{2 n-k_{0}+1} \frac{t^{n-k_{0}+1}}{n-k_{0}+1} .
\end{aligned}
$$


Therefore,

$$
\begin{aligned}
& \sum_{n=0}^{\infty} \sum_{0 \leq i_{1}, \ldots, i_{n} \leq m}\left\|\mathbb{E}\left[D_{t_{1}, \ldots, t_{n}}^{i_{1}, \ldots, i_{n}} L_{\varepsilon}(t, x)\right]\right\|_{L^{2}\left([0, T]^{n}\right)}^{2} \\
& \quad \leq \sum_{n=0}^{\infty} \sum_{k_{0}=0}^{n} \frac{t 2^{k_{0}+1}}{(\pi \sigma)^{2} k_{0} !} \Gamma\left(\frac{k_{0}+1}{2}\right)^{2} \sum_{k_{1}+\cdots+k_{m}=n-k_{0}} \frac{(4 t)^{n-k_{0}}}{k_{1} ! \cdots k_{m} !} \frac{1}{2 n-k_{0}+1} \frac{1}{n-k_{0}+1} \\
& \quad \leq \sum_{n=0}^{\infty} \sum_{k_{0}=0}^{n} \frac{2^{k_{0}+1}}{m(\pi \sigma)^{2} k_{0} !} \Gamma\left(\frac{k_{0}+1}{2}\right)^{2} \frac{(4 m t)^{n-k_{0}+1}}{\left(n-k_{0}+1\right) !} \frac{1}{2 n-k_{0}+1} \\
& \quad \leq \sum_{k_{0}=0}^{\infty} \frac{2^{k_{0}+1}}{m(\pi \sigma)^{2} k_{0} !} \Gamma\left(\frac{k_{0}+1}{2}\right)^{2} \frac{e^{m 4 t}-1}{k_{0}+1},
\end{aligned}
$$

where we have used the facts that the bound of each term of the series depends only on $k_{0}, k_{1}, \ldots, k_{m}$,

$$
\sum_{k_{1}+\cdots+k_{m}=r} \frac{1}{k_{1} ! \cdots k_{m} !}=\frac{m^{r}}{r !}
$$

and $2 n-k_{0}+1 \geq k_{0}+1$.

By Stirling formula,

$$
\frac{2^{k_{0}+1}}{\pi^{2}\left(k_{0}+1\right) !} \Gamma\left(\frac{k_{0}+1}{2}\right)^{2} \sim c k_{0}^{-3 / 2}, \quad \text { for } k_{0} \text { large. }
$$

Hence, the general term of the right-hand side of (4.22) behaves as $c k_{0}^{-3 / 2}$ and the corresponding series is convergent.

Note that in our setting $q_{0}=\cdots=q_{m}=1$, because we do not work with the $H^{(i)}$ 's but use directly the Wiener and Poisson processes.

Now, it remains to check Lemma 4.6 (i). We have

$$
\begin{aligned}
& \left\|f_{i_{1}, \ldots, i_{n}}^{\varepsilon}-f_{i_{1}, \ldots, i_{n}}\right\|_{L^{2}\left([0, T]^{n}\right)}^{2} \\
& \quad=\left\|f_{i_{1}, \ldots, i_{n}}^{\varepsilon}\right\|_{L^{2}\left([0, T]^{n}\right)}^{2}+\left\|f_{i_{1}, \ldots, i_{n}}\right\|_{L^{2}\left([0, T]^{n}\right)}^{2}-2\left\langle f_{i_{1}, \ldots, i_{n}}^{\varepsilon}, f_{i_{1}, \ldots, i_{n}}\right\rangle_{L^{2}\left([0, T]^{n}\right)} .
\end{aligned}
$$

It is clear that $f_{i_{1}, \ldots, i_{n}}^{\mathcal{E}}$ converges to $f_{i_{1}, \ldots, i_{n}}$ pointwise and using the dominated convergence theorem, we see easily that condition (i) holds.

Finally, we will show, following standard arguments, that the limit of $\int_{0}^{t} p_{\mathcal{\varepsilon}}\left(\sigma W_{s}+\right.$ $\left.\sum_{i=1}^{m} \alpha_{i} \tilde{N}_{s}^{i}-x\right) d s$, denoted by $\Lambda_{t}^{x}$, is the local time $L(t, x)$. The above estimates are uniform in $x \in \mathbb{R}$. Therefore, we can conclude that the convergence of $\int_{0}^{t} p_{\varepsilon}\left(\sigma W_{s}+\sum_{i=1}^{m} \alpha_{i} \tilde{N}_{s}^{i}-\right.$ $x) d s$ to $\Lambda_{t}^{x}$ holds in $L^{2}(\Omega \times \mathbb{R}, \mathbb{P} \otimes \mu)$, for any finite measure $\mu$. As a consequence, for any continuous function $g$ in $\mathbb{R}$ with compact support, we have that

$$
\int_{\mathbb{R}}\left(\int_{0}^{t} p_{\varepsilon}\left(\sigma W_{s}+\sum_{i=1}^{m} \alpha_{i} \tilde{N}_{s}^{i}-x\right) d s\right) g(x) d x
$$


224 A Stroock formula for Lévy processes and applications

converges in $L^{2}(\Omega)$ to $\int_{\mathbb{R}} \Lambda_{t}^{x} g(x) d x$. But, this expression also converges to

$$
\int_{0}^{t} g\left(\sigma W_{s}+\sum_{i=1}^{m} \alpha_{i} \tilde{N}_{s}^{i}\right) d s
$$

Hence,

$$
\int_{\mathbb{R}} \Lambda_{t}^{x} g(x) d x=\int_{0}^{t} g\left(\sigma W_{s}+\sum_{i=1}^{m} \alpha_{i} \tilde{N}_{s}^{i}\right) d s,
$$

which implies that $\Lambda_{t}^{x}=L(t, x)$.

\section{Chaos expansion of the price of a financial asset}

In this section, we will use the Stroock formula to get the chaotic decomposition of the asset price $S_{t}$ driven by the Lévy process $\tilde{X}_{t}=\mu t+\sigma W_{t}+\sum_{j=1}^{m} \alpha_{j} \tilde{N}_{t}^{j}$, where $\tilde{N}_{t}^{j}, j=1, \ldots, m$, are independent compensated Poisson processes with parameters $\lambda_{j}, j=1, \ldots, m$. This means that $S_{t}$ satisfies the stochastic differential equation

$$
d S_{t}=S_{t-} d \tilde{X}_{t}, \quad S_{0}=s_{0},
$$

with solution

$$
\begin{aligned}
S_{t} & =s_{0} \exp \left\{\left(\mu-\frac{\sigma^{2}}{2}\right) t-\sum_{i=1}^{m} \alpha_{i} \lambda_{i} t+\sigma W_{t}\right\} \prod_{i=1}^{m}\left(1+\alpha_{i}\right)^{N_{t}^{i}} \\
& =s_{0} \exp \left(c t+\sigma W_{t}+\sum_{i=1}^{m} \beta_{i} N_{t}^{i}\right),
\end{aligned}
$$

where $c=\mu-\sigma^{2} / 2-\sum_{i=1}^{m} \alpha_{i} \lambda_{i}$ and $\beta_{i}=\log \left(1+\alpha_{i}\right)$ for $i=1, \ldots, m$ with the condition $\alpha_{i}>-1$ to guarantee the positivity of the price (see [13]).

Remark 5.1. If $\tau$ is a jump time of the Poisson process $N^{j}$, the relative jump $\left(S_{\tau}-S_{\tau-}\right) / S_{\tau}$ is equal to $\alpha_{j}$ and the absolute jump $\log \left(S_{\tau}\right)-\log \left(S_{\tau-}\right)$ of the $\log$ price process $\log \left(S_{t}\right)$ is $\beta_{j}$.

Now we compute the iterated derivatives of $S_{t}$ and we obtain

$$
D_{s_{1}, \ldots, s_{k_{0}}}^{0, k_{0}} S_{t}=\sigma^{k_{0}} S_{t}
$$

for $s_{1}<\cdots<s_{k_{0}}<t$,

$$
D_{s}^{\ell} S_{t}=s_{0} \exp \left(-\sigma^{2} t / 2+\sigma W_{t}\right) \prod_{i=1, j \neq \ell}^{m}\left(1+\alpha_{i}\right)^{N_{t}^{i}}\left\{\left(1+\alpha_{\ell}\right)^{N_{t}^{\ell}+1}-\left(1+\alpha_{\ell}\right)^{N_{t}^{\ell}}\right\}=\alpha_{\ell} S_{t},
$$

for $s<t$, and

$$
D_{s_{1}, \ldots, s_{\ell}}^{\ell, k_{\ell}} S_{t}=\alpha_{\ell}^{k_{\ell}} S_{t}
$$


for $\ell=1, \ldots, m$. Consequently,

$$
D_{s_{1}, \ldots, s_{n}}^{i_{1}, \ldots, i_{n}} S_{t}=\sigma^{k_{0}} \alpha_{1}^{k_{1}} \cdots \alpha_{m}^{k_{m}} S_{t} \prod_{i=1}^{n} \mathbf{1}_{\left\{s_{i}<t\right\}},
$$

with $\sum_{i=0}^{m} k_{i}=n$.

Hence,

$$
\begin{aligned}
\mathbb{E}\left[D_{s_{1}, \ldots, s_{n}}^{k_{0}, \ldots, k_{m}} S_{t}\right] & =\sigma^{k_{0}} \alpha_{1}^{k_{1}} \cdots \alpha_{m}^{k_{m}} s_{0} e^{c t} \mathbb{E}_{W}\left[\exp \left(\sigma W_{t}\right)\right] \prod_{i=1}^{m} \mathbb{E}_{N_{t}^{i}}\left[\left(1+\alpha_{i}\right)^{N_{t}^{i}}\right] \prod_{k=1}^{n} \mathbf{1}_{\left\{0<s_{k}<t\right\}} \\
& =\sigma^{k_{0}} \alpha_{1}^{k_{1}} \cdots \alpha_{m}^{k_{m}} s_{0} e^{c t} \exp \left(\sigma^{2} t / 2\right) \prod_{i=1}^{m} \exp \left(\alpha_{i} \lambda_{i} t\right) \prod_{k=1}^{n} \mathbf{1}_{\left\{0<s_{k}<t\right\}}, \\
f_{k_{0}, \ldots, k_{m}}\left(s_{1}, \ldots, s_{n} ; t\right) & =s_{0} \prod_{i=0}^{m} \alpha_{i}^{k_{i}} e^{\gamma_{i} t} \mathbf{1}_{\left\{0<s_{1}<\cdots<s_{n}<t\right\}}=s_{0} e^{\mu t} \prod_{i=0}^{m} \alpha_{i}^{k_{i}} \mathbf{1}_{\left\{0<s_{1}<\cdots<s_{n}<t\right\}},
\end{aligned}
$$

with $\alpha_{0}=\sigma, \gamma_{i}=\alpha_{i} \lambda_{i}$, for $i=1, \ldots, m, \gamma_{0}=\mu-\sum_{i=1}^{m} \alpha_{i} \lambda_{i}$, and $\lambda_{0}=1$.

The chaotic decomposition of $S_{t}$ is given by

$$
S_{t}=\mathbb{E}\left[S_{t}\right]+\sum_{n=1}^{\infty} \sum_{i_{1}, \ldots, i_{n}} s_{0} e^{\mu t} \prod_{j=0}^{m} \alpha_{j}^{k_{j}} L_{n}^{i_{1}, \ldots, i_{n}}\left(\mathbf{1}_{\left\{0<s_{1}<\cdots<s_{n}<t\right\}}\right) .
$$

As the terms of the chaotic decomposition are uncorrelated, the variance has the following expression:

$$
\begin{aligned}
\operatorname{Var}\left(S_{t}\right) & =\sum_{n=1}^{\infty} \sum_{i_{1}, \ldots, i_{n}} s_{0}^{2} e^{2 \mu t} \prod_{j=0}^{m} \alpha_{j}^{2 k_{j}} \mathbb{E}\left[L_{n}^{i_{1}, \ldots, i_{n}}\left(\mathbf{1}_{\left\{0<s_{1}<\cdots<s_{n}<t\right\}}\right)^{2}\right] \\
& =\sum_{n=1}^{\infty} \sum_{i_{1}, \ldots, i_{n}} s_{0}^{2} e^{2 \mu t} \prod_{j=0}^{m}\left(\lambda_{j} \alpha_{j}^{2}\right)^{k_{j}} \frac{t^{n}}{n !} \\
& =s_{0}^{2} e^{2 \mu t} \sum_{n=1}^{\infty} \frac{t^{n}}{n !} \sum_{i_{1}, \ldots, i_{n}} \prod_{j=0}^{m}\left(\lambda_{j} \alpha_{j}^{2}\right)^{k_{j}} \\
& =s_{0}^{2} e^{2 \mu t} \sum_{n=1}^{\infty} t^{n} \sum_{k_{0}+\cdots+k_{m}=n} \prod_{j=0}^{m} \frac{\left(\lambda_{j} \alpha_{j}^{2}\right)^{k_{j}}}{k_{j} !} .
\end{aligned}
$$

By the multinomial formula,

$$
\operatorname{Var}\left(S_{t}\right)=s_{0}^{2} e^{2 \mu t} \sum_{n=1}^{\infty} \frac{\left(\sum_{j=0}^{m} t \lambda_{j} \alpha_{j}^{2}\right)^{n}}{n !}=s_{0}^{2} e^{2 t \mu}\left[e^{t \sum_{j=0}^{m} \lambda_{j} \alpha_{j}^{2}}-1\right]
$$


226 A Stroock formula for Lévy processes and applications

If we only consider the first $p$ chaos, the error of the variance is

$$
s_{0}^{2} \frac{t^{p+1}}{(p+1) !} e^{2 \mu t}\left(\sum_{j=0}^{m} \lambda_{j} \alpha_{j}^{2}\right)^{p+1} e^{\xi \sum_{j=0}^{m} \lambda_{j} \alpha_{j}^{2}}, \quad 0<\xi<t
$$

which tends to 0 if $p$ tends to infinity or $t$ tends to zero.

In the case of the Brownian motion, $\gamma_{j}=\alpha_{j}=0$ for $j=1, \ldots, m$ and

$$
\operatorname{Var}\left(S_{t}\right)=s_{0}^{2} e^{2 \mu t} \sum_{n=1}^{\infty} t^{n} \frac{\sigma^{2 n}}{n !}=s_{0}^{2} e^{2 t \mu}\left(e^{t \sigma^{2}}-1\right) .
$$

\section{Chaos expansion of the price of a European call option}

Let $U_{t}$ be the price on $t$ of a European call option written on the asset described in the last section. By the no-arbitrage theory, $U_{t}=\mathbb{E}_{\mathbb{Q}}\left[e^{-r(T-t)} g\left(S_{T}-K\right) / \mathscr{F}_{t}\right]=\mathbb{E}_{\mathbb{Q}}\left[g\left(S_{T}-K\right) / S_{t}\right]$, where $\mathbb{Q}$ is the unique martingale measure described in [6] and $g(x)=x \vee 0$. Note that in order to get the uniqueness of $\mathbb{Q}$, we have to add $m$ additional assets defined by equations

$$
d P_{t}^{j}=P_{t-}^{j} d X_{t}^{j}, \quad j=1, \ldots, m,
$$

where

$$
X_{t}^{j}=\mu_{j} t+\sigma_{j} W_{t}+\sum_{i=1}^{m} \alpha_{j, i}\left(N_{t}^{i}-\lambda_{i} t\right)
$$

with the condition that there exist constants $M, L_{1}, \ldots, L_{m}$, with $L_{j} \leq \lambda_{j}$, such that

$$
B\left(\begin{array}{c}
M \\
L_{1} \\
\vdots \\
L_{m}
\end{array}\right)=\left(\begin{array}{c}
\mu-r \\
\mu_{1}-r \\
\vdots \\
\mu_{m}-r
\end{array}\right)
$$

where $r>0$ is the fixed interest rate and $B$ is a matrix given by

$$
B=\left(\begin{array}{cccc}
\sigma & \alpha_{1} & \cdots & \alpha_{m} \\
\sigma_{1} & \alpha_{11} & \cdots & \alpha_{1 m} \\
\vdots & \vdots & & \vdots \\
\sigma_{m} & \alpha_{m 1} & \cdots & \alpha_{m m}
\end{array}\right)
$$


Using Girsanov's theorem, we find a $\mathbb{Q}$-Brownian motion $W^{\mathbb{Q}}$ and $m$ independent $\mathbb{Q}$ Poisson processes with parameters $\tilde{\lambda}_{j}=\lambda_{j}-L_{j}, N^{\mathbb{Q}, 1}, \ldots, N^{\mathbb{Q}, m}$, such that we can write

$$
\begin{aligned}
\frac{d S_{t}}{S_{t-}} & =\sigma d W_{t}^{\mathbb{Q}}+\sum_{i=1}^{m} \alpha_{i}\left(d N_{t}^{\mathbb{Q}, i}-\tilde{\lambda}_{i} d t\right)+r d t, \\
\frac{d P_{t}^{1}}{P_{t-}^{1}} & =\sigma_{1} d W_{t}^{\mathbb{Q}}+\sum_{i=1}^{m} \alpha_{1, i}\left(d N_{t}^{\mathbb{Q}, i}-\tilde{\lambda}_{i} d t\right)+r d t, \\
\vdots & \\
\frac{d P_{t}^{m}}{P_{t-}^{m}} & =\sigma_{m} d W_{t}^{\mathbb{Q}}+\sum_{i=1}^{m} \alpha_{m, i}\left(d N_{t}^{\mathbb{Q}, i}-\tilde{\lambda}_{i} d t\right)+r d t .
\end{aligned}
$$

The explicit solution of $(6.5)$ is

$$
S_{t}=s_{0} \exp \left(c t+\sigma W_{t}^{\mathbb{Q}}\right) \prod_{i=1}^{m}\left(1+\alpha_{i}\right)^{N_{t}^{\mathbb{Q}, i}}=s_{0} \exp \left(c t+\sigma W_{t}^{\mathbb{Q}}+\sum_{i=1}^{m} \beta_{i} N_{t}^{\mathbb{Q}, i}\right),
$$

where $c=r-\left(\sigma^{2} / 2\right)-\sum_{i=1}^{m} \alpha_{i} \tilde{\lambda}_{i}$.

The chaotic expansion of $U_{t}$ is given by

$$
U_{t}=\sum_{n=0}^{\infty} \sum_{i_{1}, \ldots, i_{n}} L_{n}^{i_{1}, \ldots, i_{n}}\left(f_{i_{1}, \ldots, i_{n}}(\cdot, t)\right)
$$

and using the Stroock formula, we get

$$
\begin{aligned}
f_{i_{1}, \ldots, i_{n}}\left(s_{1}, \ldots, s_{n}, t\right) & =\mathbb{E}_{\mathbb{Q}}\left[D_{s_{1}, \ldots, s_{n}}^{i_{1}, \ldots, i_{n}} U_{t}\right]=\mathbb{E}_{\mathbb{Q}}\left[D_{s_{1}, \ldots, s_{n}}^{i_{1}, \ldots, i_{n}} \mathbb{E}_{\mathbb{Q}}\left[e^{-r(T-t)} g\left(S_{T}-K\right) / \mathscr{F}_{t}\right]\right] \\
& =e^{-r(T-t)} \mathbb{E}_{\mathbb{Q}}\left[\mathbb{E}_{\mathbb{Q}} D_{s_{1}, \ldots, s_{n}}^{i_{1}, \ldots, i_{n}}\left[g\left(S_{T}-K\right) \mathbf{1}_{\left\{s_{1}<\cdots<s_{n}<t\right\}} / \mathscr{F}_{t}\right]\right] \\
& =\mathbb{E}_{\mathbb{Q}}\left[D_{s_{1}, \ldots, s_{n}}^{i_{1}, \ldots, i_{n}} g\left(S_{T}-K\right)\right] e^{-r(T-t)} \mathbf{1}_{\left\{0<s_{1}<\cdots<s_{n}<t\right\}},
\end{aligned}
$$

where we have used the known commutative property between the derivative operator and the conditional expectation (see [10]).

Now, we have to compute $\mathbb{E}_{\mathbb{Q}}\left[D_{s_{1}, \ldots, s_{n}}^{i_{1}, \ldots, i_{n}} g\left(S_{T}-K\right)\right]$. In order to do this, we approximate the function $g$, uniformly, by the sequence of $\mathscr{C}^{\infty}$ functions:

$$
g_{\varepsilon}(x)=\int_{-\infty}^{x} \Phi_{\varepsilon}(y) d y
$$

where $\Phi_{\varepsilon}$ is the cumulative probability function of the centered normal law with variance $\varepsilon$.

Observe that the first derivative of $g_{\varepsilon}$ is $\Phi_{\varepsilon}$ and the high-order derivatives are the functions $p_{\varepsilon}^{(j-2)}(x)$ for $j \geq 2$, that are for every $j$, the $(j-2)$-order derivatives of the centered Gaussian kernel of variance $\varepsilon$, denoted by $p_{\varepsilon}$. 
228 A Stroock formula for Lévy processes and applications

Let $F_{\varepsilon}=g_{\varepsilon}\left(S_{T}-K\right)$. Clearly, $F_{\varepsilon}$ converges in $L^{2}$ to $F=\left(S_{T}-K\right)^{+}=U_{T}$. Then, applying Remark 2.8 to $g_{\varepsilon}$ and assuming $s_{1}<s_{2}<\cdots<s_{n}<T$,

$$
\begin{aligned}
D_{\mathcal{S}_{1}, \ldots, s_{n}}^{i_{1}, \ldots, i_{n}} F_{\varepsilon} & =D_{J_{0}}^{0, k_{0}} D_{J_{1}}^{1, k_{1}} \cdots D_{J_{m}}^{m, k_{m}} F_{\mathcal{\varepsilon}} \\
& =\sum_{i=1}^{m} \sum_{\ell_{i}=0}^{k_{i}} \prod_{j=1}^{m}\left(\begin{array}{c}
k_{j} \\
\ell_{j}
\end{array}\right)(-1)^{n-k_{0}-\sum_{j=1}^{m} \ell_{j}} \partial_{W}^{\left(k_{0}\right)} g_{\varepsilon}\left(\widetilde{S}_{T}-K\right),
\end{aligned}
$$

where $\widetilde{S}_{T}=S_{T} e^{\sum_{j=1}^{m} \beta_{j} \ell_{j} T}$.

Using the formula $f\left(a e^{b x}-c\right)^{(n)}=\sum_{j=1}^{n} f^{(j)}\left(a e^{b x}-c\right) b^{n} a^{j} e^{j b x} c_{n, j}$ with $c_{n, n}=c_{n, 1}=1$, we get the following.

(i) If $k_{0}=0$,

$$
D_{S_{1}, \ldots, s_{n}}^{i_{1}, \ldots, i_{n}} F_{\varepsilon}=\sum_{i=1}^{m} \sum_{0 \leq \ell_{i} \leq k_{i}}(-1)^{n-\sum_{i=1}^{m} \ell_{i}} \prod_{i=1}^{m}\left(\begin{array}{c}
k_{i} \\
\ell_{i}
\end{array}\right) g_{\mathcal{\varepsilon}}\left(\tilde{S}_{T}-K\right) .
$$

(ii) If $k_{0}>0$,

$$
D_{s_{1}, \ldots, s_{n}}^{i_{1}, \ldots, i_{n}} F_{\varepsilon}=\sum_{i=1}^{m} \sum_{0 \leq \ell_{i} \leq k_{i}}(-1)^{n-k_{0}-\sum_{i=1}^{m} \ell_{i}} \prod_{i=1}^{m}\left(\begin{array}{c}
k_{i} \\
\ell_{i}
\end{array}\right) \sigma^{k_{0}} \sum_{j=1}^{k_{0}} g_{\varepsilon}^{(j)}\left(\tilde{S}_{T}-K\right) \tilde{S}_{T}^{j} c_{k_{0}, j} .
$$

Therefore, we have to compute and pass to limit, when $\varepsilon$ tends to zero, three types of expectations:

$$
\begin{gathered}
\mathbb{E}\left[g_{\varepsilon}\left(\tilde{S}_{T}-K\right)\right], \\
\mathbb{E}\left[\Phi_{\epsilon}\left(\tilde{S}_{T}-K\right) \tilde{S}_{T}\right], \\
\mathbb{E}\left[p_{\varepsilon}^{(\ell)}\left(\tilde{S}_{T}-K\right) \tilde{S}_{T}^{\ell+2}\right]
\end{gathered}
$$

for $\ell \geq 0$.

In the first case, using the uniform convergence of $g_{\varepsilon}$ to $g$, we obtain as a limit

$$
\mathbb{E}\left[g\left(\tilde{S}_{T}-K\right)\right]=\mathbb{E}\left[\left(\tilde{S}_{T}-K\right)^{+}\right]=\mathbb{E}\left[\tilde{S}_{T} \mathbf{1}_{\left\{\tilde{S}_{T}>K\right\}}\right]-K \mathbb{P}\left\{\tilde{S}_{T}>K\right\}
$$

For the second case, using the dominated convergence theorem, we have that the limit is

$$
\mathbb{E}\left[\tilde{S}_{T} \mathbf{1}_{\left\{\tilde{S}_{T}>K\right\}}\right] .
$$

So for the two first expectations, the problem reduces to compute explicitly $\mathbb{E}\left[\widetilde{S}_{T} \mathbf{1}_{\left\{\tilde{S}_{T}>K\right\}}\right]$ and $\mathbb{P}\left\{\tilde{S}_{T}>K\right\}$.

Fixing Poisson processes, we define

$$
C=s_{0} \exp \left\{\sum_{j=1}^{m} \beta_{j} N_{T}^{(j)}+\left(r-\frac{\sigma^{2}}{2}+\sum_{j=1}^{m}\left(\beta_{j} \ell_{j}-\alpha_{j} \tilde{\lambda}_{j}\right)\right) T\right\} .
$$


In order to get the expectations with respect to the Gaussian part, we need only the fact that

$$
\mathbb{P}_{W_{T}}\left\{e^{\sigma W_{T}} \geq \gamma\right\}=1-\Phi\left(\frac{\log (\gamma)}{\sigma \sqrt{T}}\right)
$$

and the following lemma.

Lemma 6.1. Let $\gamma \geq 0$. Then

$$
\mathbb{E}_{W_{T}}\left[e^{\sigma W_{T}} \mathbf{1}_{\left\{e^{\left.\sigma W_{T}>\gamma\right\}}\right.}\right]=e^{\sigma^{2} T / 2} \Phi\left(\sigma \sqrt{T}-\frac{\log (\gamma)}{\sigma \sqrt{T}}\right) .
$$

Proof. We have

$$
\begin{aligned}
\mathbb{E}_{W_{T}}\left[e^{\sigma W_{T}} \mathbf{1}_{\left\{e^{\left.\sigma W_{T}>\gamma\right\}}\right.}\right] & =\int_{\mathbb{R}} e^{x \sigma} \mathbf{1}_{\left\{e^{x \sigma}>\gamma\right\}} \exp \left(-\frac{x^{2}}{2 T}\right) \frac{d x}{\sqrt{2 \pi T}} \\
& =\int_{(\log \gamma) / \sigma}^{\infty} \exp \left(-\frac{x^{2}}{2 T}+\sigma x\right) \frac{d x}{\sqrt{2 \pi T}} \\
& =e^{\sigma^{2} T / 2} \int_{(\log \gamma) / \sigma \sqrt{T}}^{\infty} \exp \left(-\frac{(y-\sigma \sqrt{T})^{2}}{2}\right) \frac{d y}{\sqrt{2 \pi}} \\
& =e^{\sigma^{2} T / 2} \int_{((\log \gamma) / \sigma \sqrt{T})-\sigma \sqrt{T}}^{\infty} \exp \left(-\frac{x^{2}}{2}\right) \frac{d x}{\sqrt{2 \pi}} .
\end{aligned}
$$

Finally, for the expectations of third type, we need the following result.

Lemma 6.2.

$$
\lim _{\varepsilon \rightarrow 0} \mathbb{E}_{W_{T}}\left[p_{\varepsilon}^{(\ell)}\left(C e^{\sigma W_{T}}-K\right)\left(C e^{\sigma W_{T}}\right)^{\ell+2}\right]=(-1)^{\ell} h_{\ell}^{(\ell)}(K),
$$

where

$$
h_{\ell}(x)=x^{\ell+2} f(x)=x^{\ell+1} p_{\sigma \sqrt{T}}\left(\log \left(\frac{x}{C}\right)\right)
$$

with $f$ being the density of the log-normal law with parameters $\log (C)$ and $\sigma \sqrt{T}$.

Proof. We have

$$
\mathbb{E}_{W_{T}}\left[p_{\varepsilon}^{(\ell)}\left(C e^{\sigma W_{T}}-K\right)\left(C e^{\sigma W_{T}}\right)^{\ell+2}\right]=\int_{\mathbb{R}} p_{\varepsilon}^{(\ell)}(x-K) x^{\ell+2} f(x) d x .
$$

Taking $h_{\ell}(x)=x^{\ell+2} f(x)$ and integrating by parts, using that $h_{\ell}$ and all its derivatives are fast decreasing functions, the last integral is equal to

$$
(-1)^{\ell} \int_{\mathbb{R}} p_{\varepsilon}(x-K) h_{\ell}^{(\ell)}(x) d x=(-1)^{\ell}\left(p_{\varepsilon} * h_{\ell}^{(\ell)}\right)(K),
$$


where $*$ denotes the convolution. Using that $p_{\varepsilon}$ is an approximation of the identity, we obtain the limit

$$
(-1)^{\ell} h_{\ell}^{(\ell)}(K)
$$

As a conclusion, we have

$$
\begin{aligned}
f_{i_{1}, \ldots, i_{n}}\left(s_{1}, \ldots, s_{n} ; t\right) & \\
= & \sum_{\ell_{1}=0}^{k_{1}} \cdots \sum_{\ell_{m}=0}^{k_{m}}(-1)^{n-k_{0}-\ell_{1}-\cdots-\ell_{m}}\left(\begin{array}{c}
k_{1} \\
\ell_{1}
\end{array}\right) \cdots\left(\begin{array}{c}
k_{m} \\
\ell_{m}
\end{array}\right) \sigma^{k_{0}} \\
& \times \sum_{j_{1}, \ldots, j_{m}=0}^{\infty} e^{-\tilde{\lambda}_{1} T} \cdots e^{-\tilde{\lambda}_{m} T} \frac{\tilde{\lambda}_{1}^{j_{1}} \cdots \tilde{\lambda}_{m}^{j_{m}} T^{j_{1}+\cdots+j_{m}}}{j_{1} ! \cdots j_{m} !} \\
& \times\left[\left(C\left(j_{1}, \ldots, j_{m}\right) e^{\left(\sigma^{2} T\right) / 2}-K \mathbf{1}_{\left\{k_{0}=0\right\}}\right) \Phi\left(\frac{\log C\left(j_{1}, \ldots, j_{m}\right)-\log K}{\sigma \sqrt{T}}\right)\right. \\
& \left.\quad+\sum_{l=0}^{k_{0}-2} c_{k_{0}, l+2}(-1)^{l} h_{l}^{(l)}(K) \mathbf{1}_{\left\{k_{0} \geq 2\right\}}\right] e^{-r(T-t)} \mathbf{1}_{\left\{0<s_{1}<\cdots<s_{n}<t\right\}},
\end{aligned}
$$

where

$$
\begin{gathered}
h_{l}(x)=x^{l+1} p_{\sigma \sqrt{T}}\left(\log x-\log C\left(j_{1}, \ldots, j_{m}\right)\right), \\
C\left(j_{1}, \ldots, j_{m}\right)=s_{0} \exp \left\{\sum_{i=1}^{m} \beta_{i} j_{i}+\left(r-\frac{\sigma^{2}}{2}+\sum_{i=1}^{m}\left(\beta_{i} \ell_{i}-\alpha_{i} \tilde{\lambda}_{i}\right)\right) T\right\} .
\end{gathered}
$$

In the case of no jumps in the model, we obtain

$$
\begin{aligned}
f_{n}\left(s_{1}, \ldots, s_{n} ; t\right)=\sigma^{n}[ & \left(S_{0} e^{r T}-K \mathbf{1}_{\{n=0\}}\right) \Phi\left(\frac{\log C-\log K}{\sigma \sqrt{T}}\right) \\
& \left.+\sum_{l=0}^{n-2} c_{n, l+2}(-1)^{l} h_{l}^{(l)}(K) \mathbf{1}_{\{n \geq 2\}}\right] e^{-r(T-t)} \mathbf{1}_{\left\{0<s_{1}<\cdots<s_{n}<t\right\}},
\end{aligned}
$$

where now $C$ reduces to $s_{0} \exp \left\{\left(r-\left(\sigma^{2} / 2\right)\right) T\right\}$.

\section{Chaos expansion of the tracking error in discrete delta-neutral hedging}

Assume that we are in a neutral risk environment. That is, from now on, we are concerned with the unique probability $\mathbb{Q}$ that makes $e^{-r t} S_{t}$ a martingale. Here we follow some ideas from [5].

Let $U_{t}$ be the prize of a derivative security. Its actualized price $\tilde{U}_{t}$ is given by

$$
e^{-r t} U_{t}=E\left[e^{-r T} h\left(S_{T}\right) / \mathscr{F}_{t}\right] .
$$

By the Markov property of $S_{t}$, the process $\tilde{U}_{t}$ can be written as a function of $t$ and $S_{t}$. Moreover thanks to (6.6), we can write $\tilde{U}_{t}=u\left(t, Y_{t}\right)$, where $Y_{t}=c t+\sigma W_{t}^{\mathbb{Q}}+\sum_{i=1}^{m} \beta_{i} N_{t}^{\mathbb{Q}, i}$ and $\beta_{i}=\ln \left(1+\alpha_{i}\right)$. By its definition, $\tilde{U}_{t}$ is a martingale. 
We set

$$
u^{1}\left(s, Y_{s^{-}}, y\right):=u\left(s, Y_{s^{-}}+y\right)-u\left(s, Y_{s^{-}}\right)-\frac{\partial u}{\partial x}\left(s, Y_{s^{-}}\right) y
$$

If the function $u$ is regular, the Itô formula (see Protter [13, pages 81-82]) applied to $u\left(t, Y_{t}\right)$ yields

$$
\begin{aligned}
u\left(t, Y_{t}\right)-u\left(0, Y_{0}\right)= & \int_{0}^{t}\left(\frac{\partial u}{\partial s}\left(s, Y_{s}\right)+c \frac{\partial u}{\partial x}\left(s, Y_{s}\right)\right) d s+\frac{1}{2} \int_{0}^{t} \sigma^{2} \frac{\partial^{2} u}{\partial x^{2}}\left(s, Y_{s}\right) d s \\
& +\int_{0}^{t} \sigma \frac{\partial u}{\partial x}\left(s, Y_{s-}\right) d W_{s}^{\mathbb{Q}}+\sum_{j=1}^{m} \beta_{j} \int_{0}^{t} \frac{\partial u}{\partial x}\left(s, Y_{s-}\right) d N_{s}^{\mathbb{Q}, j} \\
& +\sum_{0<s \leq t} u^{1}\left(s, Y_{s-}, \Delta Y_{s}\right) .
\end{aligned}
$$

Now, [9, Lemma 5, page 773] due to Nualart and Schoutens (2001) applied to $u^{1}\left(s, Y_{s-}, y\right)$ gives

$$
\begin{aligned}
\sum_{0<s \leq t} u^{1}\left(s, Y_{s-}, \Delta Y_{s}\right)= & \sum_{i=1}^{m+1} \int_{0}^{t}\left(\int_{\mathbb{R}} u^{1}\left(s, Y_{s^{-}}, y\right) p_{i}(y) v(d y)\right) d H_{s}^{(i)} \\
& +\int_{0}^{t} \int_{\mathbb{R}} u^{1}\left(s, Y_{s^{-}}, y\right) v(d y) d s,
\end{aligned}
$$

where $p_{i}(y)=a_{i, i} x^{i}+a_{i, i-1} x^{i-1}+\cdots+a_{i, 1} x$. Note that there are only $m+1$ processes $H^{i}$ associated to $Y$ which are different from zero (see [6]).

In our case the explicit expression of the right-hand side of the last equation is a martingale plus the following term:

$$
\int_{0}^{t} \sum_{j=1}^{m}\left(\tau_{\beta_{j}} u\left(s, Y_{s-}\right)-\frac{\partial u}{\partial x}\left(s, Y_{s-}\right) \beta_{j}\right) \tilde{\lambda}_{j} d s
$$

where $\tau_{\beta} u(t, x)=u(t, x+\beta)-u(t, x)$.

Hence, the martingale property of $u\left(t, Y_{t}\right)$ is equivalent to the condition that $u$ satisfies the following partial differential integral equation:

$$
\begin{gathered}
\frac{\partial u}{\partial t}(t, x)+c \frac{\partial u}{\partial x}(t, x)+\frac{\sigma^{2}}{2} \frac{\partial^{2} u}{\partial x^{2}}(t, x)+\sum_{j=1}^{m} \tilde{\lambda}_{j} \tau_{\beta_{j}} u(t, x)=0, \\
u(0, x)=u_{0}(x)
\end{gathered}
$$

where $u_{0}$ is a given function. 
232 A Stroock formula for Lévy processes and applications

Moreover $\partial_{x}^{n-j} \circ \tau_{\beta_{i_{1}}} \circ \ldots \circ \tau_{\beta_{i}} u\left(t, Y_{t}\right), i_{1}, \ldots, i_{j} \in\{1,2, \ldots, m\}$ and $j \in\{0,1, \ldots, n\}$, is also a martingale since $\partial_{x}^{n-j} \circ \tau_{\beta_{i_{1}}} \circ \cdots \circ \tau_{\beta_{i}} u(t, x)$ satisfy (7.6).

Being $u\left(t, Y_{t}\right)$ a functional assumed to be square integrable, by the Stroock formula, its chaos expansion is

$$
\begin{aligned}
& u\left(t, Y_{t}\right) \\
& =\sum_{n=0}^{\infty} \sum_{i_{1}, \ldots, i_{n}} J_{n}^{i_{1}, \ldots, i_{n}}\left(E\left[D_{s_{1}, \ldots, s_{n}}^{i_{1}, \ldots, i_{n}} u\left(t, Y_{t}\right)\right]\right) \\
& =\sum_{n=0}^{\infty} \sum_{i_{1}, \ldots, i_{n}, k_{0}+\cdots+k_{m}=n} E\left[\sigma^{k_{0}} \partial_{x}^{k_{0}} \circ \tau_{\beta_{1}}^{k_{1}} \circ \cdots \circ \tau_{\beta_{m}}^{k_{m}} u\left(t, Y_{t}\right)\right] J_{n}^{i_{1}, \ldots, i_{n}}\left(\mathbf{1}_{\left\{0<s_{1}<\cdots<s_{n} \leq t\right\}}\right) \\
& =\sum_{n=0}^{\infty} \sum_{i_{1}, \ldots, i_{n}} \sigma^{k_{0}} \partial_{x}^{k_{0}} \circ \tau_{\beta_{1}}^{k_{1}} \circ \cdots \circ \tau_{\beta_{m}}^{k_{m}} u(0,0) J_{n}^{i_{1}, \ldots, i_{n}}\left(\mathbf{1}_{\left\{0<s_{1}<\cdots<s_{n} \leq t\right\}}\right) .
\end{aligned}
$$

From the Clark-Ocone formula, we have

$$
e^{r T} U_{0}-U_{T}+\int_{0}^{T} \psi_{0}(s) d \tilde{S}(s)+\sum_{j=1}^{m} \int_{0}^{T} \psi_{j}(s) d \widetilde{P}_{j}(s)=0
$$

with

$$
\begin{gathered}
\left(\psi_{0}(s), \ldots, \psi_{m}(s)\right)=\left(\phi_{0}(s), \ldots, \phi_{m}(s)\right) B^{-1}\left(\begin{array}{cccc}
\frac{1}{S^{*}(s-)} & 0 & \ldots & 0 \\
0 & \frac{1}{P_{1}^{*}(s-)} & \ldots & 0 \\
\vdots & \vdots & & \vdots \\
0 & 0 & \cdots & \frac{1}{P_{m}^{*}(s-)}
\end{array}\right), \\
\phi_{j}(s)=E\left[D_{s}^{(j)} e^{-r T} U_{T} / \mathscr{F}_{s-}\right], \quad j=0, \ldots, m .
\end{gathered}
$$

It represents the value of a dynamic portfolio with a short position in one derivative and a suitable large position on the risky assets.

Let $\left\{t_{0}=0<t_{1}<\cdots<t_{p}=T\right\}$ be a partition of $[0, T]$ such that $t_{j+1}-t_{j}=\delta$ for all $j=0, \ldots, p-1$, and hence $p \delta=T$.

Discrete hedging corresponds to the value process

$$
e^{r T} U_{0}-U_{T}+\sum_{i=0}^{p-1} \int_{t_{i}}^{t_{i+1}} \psi_{0}\left(t_{i}\right) d \widetilde{S}(s)+\sum_{j=1}^{m} \sum_{i=0}^{p-1} \int_{t_{i}}^{t_{i+1}} \psi_{j}\left(t_{i}\right) d \widetilde{P}_{j}(s),
$$


and the difference between values of the two portfolios is the so-called tracking error that can be written in this case as

$$
\begin{aligned}
E_{T}= & \sum_{j=0}^{p-1} \int_{t_{j}}^{t_{j+1}} e^{-r T}\left(E\left[D_{s}^{0} U_{T} / \mathscr{F}_{s}\right]-E\left[D_{t_{j}}^{0} U_{T}\right] / \mathscr{F}_{t_{j}}\right) d W_{s}^{\mathbb{Q}} \\
& +\sum_{k=1}^{m} \sum_{j=0}^{p-1} \int_{t_{j}}^{t_{j+1}} e^{-r T}\left(E\left[D_{s}^{k} U_{T} / \mathscr{F}_{s}\right]-E\left[D_{t_{j}}^{k} U_{T}\right] / \mathscr{F}_{t_{j}}\right) d \tilde{N}_{s}^{\mathbb{Q}, k} .
\end{aligned}
$$

Of course, this is a centered random variable that converges to zero when the partition becomes finer and finer but, moreover, it has no first chaos. In fact

$$
E_{T}=\sum_{n=2}^{\infty} \sum_{i_{1}, \ldots, i_{n}} \sigma^{k_{0}} \partial_{x}^{k_{0}} \circ \tau_{\beta_{1}}^{k_{1}} \circ \cdots \circ \tau_{\beta_{m}}^{k_{m}} u(0,0) J_{n}^{i_{1}, \ldots, i_{n}}\left(1-\sum_{j=0}^{p-1} \mathbf{1}_{\left\{s_{n-1<t_{j}<s_{n} \leq t_{j+1}}\right\}}\right) .
$$

Then

$$
\operatorname{Var}\left(E_{T}\right)=\sum_{n=2}^{\infty} \sum_{i_{1}, \ldots, i_{n}}\left[\sigma^{k_{0}} \partial_{x}^{k_{0}} \circ \tau_{\beta_{1}}^{k_{1}} \circ \cdots \circ \tau_{\beta_{m}}^{k_{m}} u(0,0)\right]^{2} \int_{0}^{T} \int_{0}^{s_{n}} \cdots \int_{0}^{s_{2}} \mathbf{1}_{B_{n}(\pi)} d s_{1} \cdots d s_{n},
$$

where $B_{n}(\pi)$ is the set of $n$-tuples $\left(s_{1}, \ldots, s_{n}\right)$ such that $s_{n-1}$ and $s_{n}$ become in the same interval of the partition. On one hand,

$$
\operatorname{Var}\left(E_{T}\right) \leq \sum_{n=2}^{\infty} \sum_{i_{1}, \ldots, i_{n}}\left[\sigma^{k_{0}} \partial_{x}^{k_{0}} \circ \tau_{\beta_{1}}^{k_{1}} \circ \cdots \circ \tau_{\beta_{m}}^{k_{m}}(u)(0,0)\right]^{2} \frac{T^{n}}{n !} .
$$

On the other hand, it is easy to see that

$$
\begin{aligned}
\int_{0}^{T} \int_{0}^{s_{n}} \cdots \int_{0}^{s_{2}} \mathbf{1}_{B_{n}(\pi)} d s_{1} \cdots d s_{n} & =\sum_{j=0}^{p-1}\left((j+1)^{n}-j^{n}-n j^{n-1}\right) \frac{\delta^{n}}{n !} \\
& =P_{n-1}(p) \frac{\delta^{n}}{n !},
\end{aligned}
$$

where $P_{n-1}$ denotes a polynomial with degree $n-1$. Hence, $\operatorname{Var}\left(E_{T}\right)=O(\delta)$.

Under the historical probability, $W_{t}=W_{t}^{\mathbb{Q}}-v_{0} t$ is a Brownian motion and $\tilde{N}_{t}^{i}=$ $\tilde{N}_{t}^{\mathbb{Q}, i}-v_{i} t$, for $i=1, \ldots, m$, are Poisson processes, where $\nu_{0}=(\mu-r) / \sigma$ and $\nu_{i}=\left(\left(\lambda_{i}-\right.\right.$ $\left.\left.\tilde{\lambda}_{i}\right)\left(\beta_{i}-\alpha_{i}\right) / \beta_{i}\right)$.

If we define $Z_{t}=Y_{t}-\gamma t=c t+\sigma W_{t}+\sum_{i=1}^{m} \beta_{i} N_{t}^{i}$, with $\gamma=\sigma v_{o}+\sum_{i=1}^{m} \beta_{i} \nu_{i}$, then we can write $u\left(t, Y_{t}\right)=u\left(t, Z_{t}+\gamma t\right)$. Note that $u\left(t, Z_{t}\right)$ is a $\mathbb{P}$-martingale since $u(t, x)$ satisfies (7.6) but $u\left(t, Z_{t}+\gamma t\right)$ is not.

Using the classical Taylor formula on $u\left(t, Z_{t}+\gamma t\right)$, we obtain

$$
u\left(t, Z_{t}+\gamma t\right)=\sum_{\ell=0}^{\infty} \frac{1}{\ell !} \partial^{\ell} u\left(t, Z_{t}\right) \gamma^{\ell} t^{\ell} .
$$




\section{A Stroock formula for Lévy processes and applications}

Therefore, the chaos expansion of the tracking error becomes

$$
E_{T}=\sum_{n=2}^{\infty} \sum_{i_{1}, \ldots, i_{n}} \sum_{\ell=0}^{\infty} \frac{\gamma^{\ell} T^{\ell}}{\ell !} \sigma^{k_{0}} \partial_{x}^{k_{0}+\ell} \circ \tau_{\beta_{1}}^{k_{1}} \circ \cdots \circ \tau_{\beta_{m}}^{k_{m}} u(0,0) J_{n}^{i_{1}, \ldots, i_{n}}\left(\mathbf{1}_{B_{n}(\pi)}\right)
$$

and has the same behavior as before.

\section{Acknowledgments}

This paper was written during a visit of M'hamed Eddahbi to the Centre de Recerca Matemàtica (IEC), Universitat Autònoma de Barcelona, Barcelona. This author would like to express his sincere thanks to the CRM and the research group en Anàlisi Estocàstica for generous support and hospitality. He was also supported by the Spanish Ministerio de Educación, Cultura y Deporte, Grant SB2000-0060. The second and third authors were supported by the Spanish Ministerio de Educación, Cultura y Deporte, Grant BFM20000009 and by the Catalan CIRIT, Grant 2001SGR00174. The third author expresses his thanks to the Laboratoire de Probabilités de l'Université Paul Sabatier, Toulouse, where part of this work was completed during a visit.

\section{References}

[1] M. T. Barlow, Necessary and sufficient conditions for the continuity of local time of Lévy processes, Ann. Probab. 16 (1988), no. 4, 1389-1427.

[2] J. Bertoin, Lévy Processes, Cambridge Tracts in Mathematics, vol. 121, Cambridge University Press, Cambridge, 1996.

[3] L. Coutin, D. Nualart, and C. A. Tudor, Tanaka formula for the fractional Brownian motion, Stochastic Process. Appl. 94 (2001), no. 2, 301-315.

[4] M. Eddahbi, R. Lacayo, J. L. Solé, J. Vives, and C. A. Tudor, Regularity of the local time for the d-dimensional fractional Brownian motion with $N$-parameters, Stochastic Anal. Appl. 23 (2005), no. 2, 383-400.

[5] V. Lacoste, Wiener chaos: a new approach to option hedging, Math. Finance 6 (1996), no. 2, 197-213.

[6] J. A. León, J. L. Solé, F. Utzet, and J. Vives, On Lévy processes, Malliavin calculus and market models with jumps, Finance Stoch. 6 (2002), no. 2, 197-225.

[7] J. A. León, J. L. Solé, and J. Vives, The chaos decomposition of functionals of the jump times for the Poisson process over a compact time interval, Stochastic Models (Spanish) (Guanajuato, 1998), Aportaciones Mat. Investig., vol. 14, Sociedad Matemática Mexicana, México, 1998, pp. 269-282.

[8] D. Nualart and W. Schoutens, Chaotic and predictable representations for Lévy processes, Stochastic Process. Appl. 90 (2000), no. 1, 109-122.

[9] __ Backward stochastic differential equations and Feynman-Kac formula for Lévy processes, with applications in finance, Bernoulli 7 (2001), no. 5, 761-776.

[10] D. Nualart and J. Vives, Anticipative calculus for the Poisson process based on the Fock space, Séminaire de Probabilités, XXIV, 1988/89, Lecture Notes in Math., vol. 1426, SpringerVerlag, Berlin, 1990, pp. 154-165.

[11] Chaos expansions and local times, Publ. Mat. 36 (1992), no. 2B, 827-836.

[12] N. Privault, Distribution-valued iterated gradient and chaotic decompositions of Poisson jump times functionals, Publ. Mat. 46 (2002), no. 1, 27-48. 
[13] P. E. Protter, Stochastic Integration and Differential Equations, 2nd ed., Applications of Mathematics (New York), vol. 21, Springer-Verlag, Berlin, 2004.

[14] K. Sato, Lévy Processes and Infinitely Divisible Distributions, Cambridge Studies in Advanced Mathematics, vol. 68, Cambridge University Press, Cambridge, 1999.

M. Eddahbi: Département de Mathématiques et Infomatique, Faculté des Sciences et Techniques (FSTG), Université Cadi Ayyad, BP 549, Marrakech, Morocco

E-mail address: eddahbi@fstg-marrakech.ac.ma

J. L. Solé: Departament de Matemàtiques, Universitat Autònoma de Barcelona, 08193 Bellaterra, Barcelona, Spain

E-mail address: jllsole@mat.uab.es

J. Vives: Departament de Matemàtiques, Universitat Autònoma de Barcelona, 08193 Bellaterra, Barcelona, Spain

E-mail address: vives@mat.uab.es 


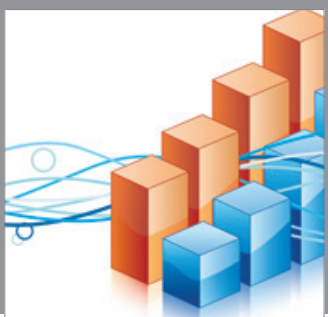

Advances in

Operations Research

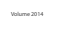

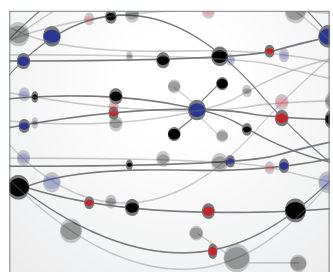

\section{The Scientific} World Journal
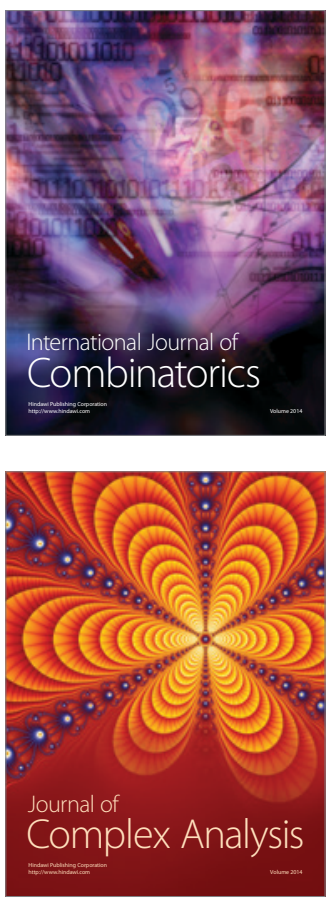

International Journal of

Mathematics and

Mathematical

Sciences
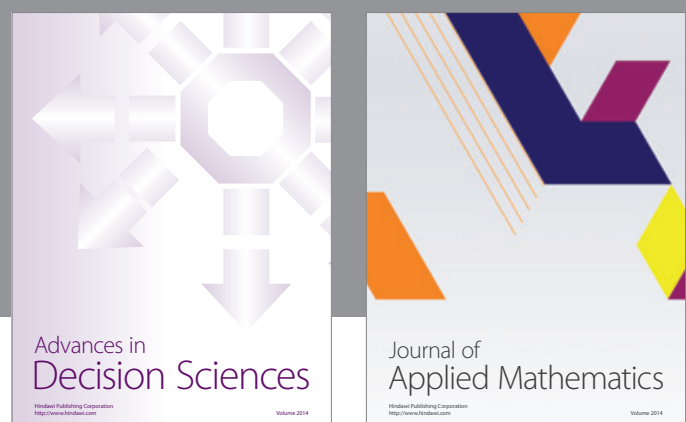

Journal of

Applied Mathematics
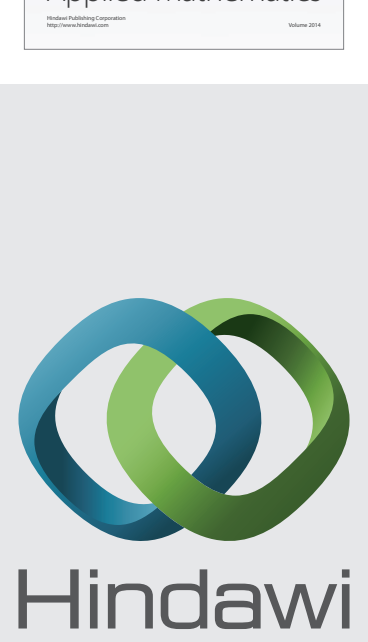

Submit your manuscripts at http://www.hindawi.com
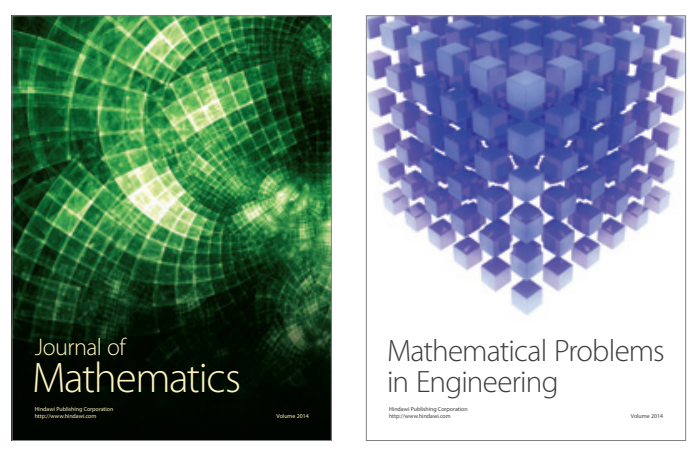

Mathematical Problems in Engineering
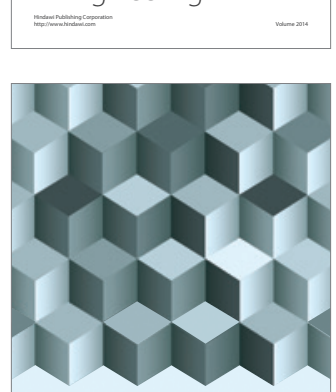

Journal of

Function Spaces
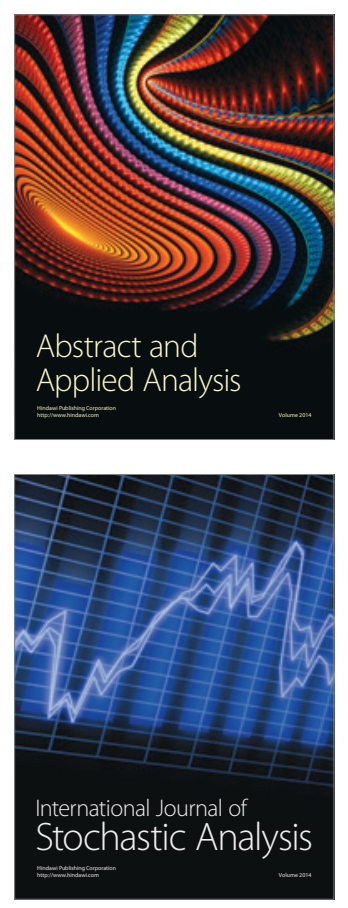

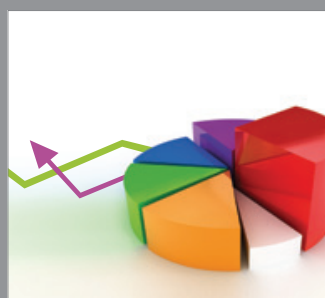

ournal of

Probability and Statistics

Promensencen
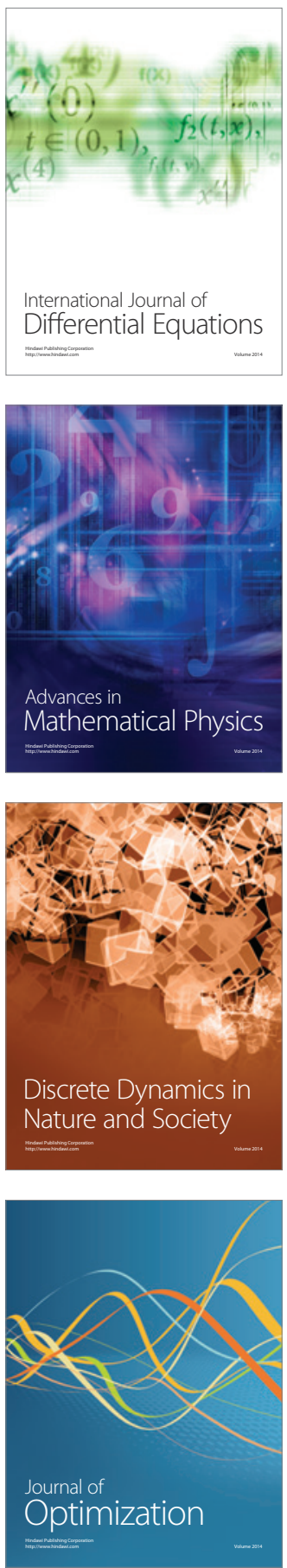